TI 2011-086/1

Tinbergen Institute Discussion Paper

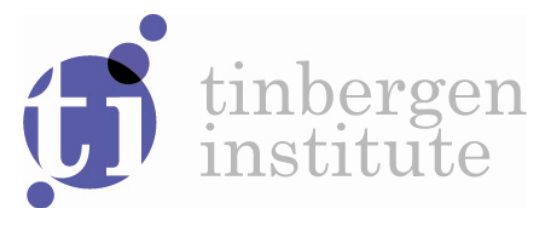

\title{
New Characterizations for Largeness of the Core
}

Arantza Estévez-Fernández

Department of Econometrics and Operations Research, VU University Amsterdam, and Tinbergen Institute. 
Tinbergen Institute is the graduate school and research institute in economics of Erasmus University Rotterdam, the University of Amsterdam and VU University Amsterdam.

More TI discussion papers can be downloaded at http://www.tinbergen.nl

Tinbergen Institute has two locations:

Tinbergen Institute Amsterdam

Gustav Mahlerplein 117

1082 MS Amsterdam

The Netherlands

Tel.: +31(0)205251600

Tinbergen Institute Rotterdam

Burg. Oudlaan 50

3062 PA Rotterdam

The Netherlands

Tel.: +31(0)10 4088900

Fax: +31(0)104089031

Duisenberg school of finance is a collaboration of the Dutch financial sector and universities, with the ambition to support innovative research and offer top quality academic education in core areas of finance.

DSF research papers can be downloaded at: http://www.dsf.nl/

Duisenberg school of finance

Gustav Mahlerplein 117

1082 MS Amsterdam

The Netherlands

Tel.: +31(0)20 5258579 


\title{
New characterizations for largeness of the core
}

\author{
Arantza Estévez-Fernández ${ }^{a}$
}

\begin{abstract}
:
In this paper we provide three new characterizations of largeness of the core. The first characterization of largeness of the core is based on minimal covers of the grand coalition and associated inequalities. The second characterization shows the relation between the bases that provide core elements of the game and the bases that provide core elements of the games that are obtained from the original one by increasing the value of the grand coalition. The third characterization of largeness of the core is based on the idea that if a base of the grand coalition does not provide a core element of the game, it should not provide a core element of a game which differs from the original one only by an increase of the value of the grand coalition. Based on these new characterizations, we show the equivalence between largeness of the core and stability of the core for games with at most 5 players and we revise some results on the literature concerning largeness of the core.
\end{abstract}

Keywords: core, upper core, largeness of the core, exactness.

JEL classification: C71.

\section{Introduction}

Cooperative (transferable utility) game theory analyzes the problem of sharing a common reward or cost that has been obtained by means of cooperation among a group of agents. In order to study this type of sharing problems, a cooperative game is introduced. A cooperative game is defined by the set of players and a characteristic function that assigns to each possible group of players (or coalition) a real value representing the total profit that this coalition can obtain by means of cooperation. This type of problem was first analyzed within the framework of cooperative game theory in Von Neumann and Morgenstern (1944). In Von Neumann and Morgenstern (1944), the stable set is put forward as a solution concept for this type of problems. The stable set of a game is the set of individually rational allocations which are both internally

\footnotetext{
aTinbergen Institute and Department of Econometrics and Operations Research, VU University Amsterdam, De Boelelaan 1105, 1081HV Amsterdam, The Netherlands. E-mail address: arantza.estevezfernandez@vu.nl
} 
and externally stable. In other words, the stable set is the set of allocations for which each player gets at least his standalone value, no allocation in the stable set is dominated by another allocation in the stable set, and all allocations outside the stable set are dominated by elements in the stable set. The stable set can be seen as a non-cooperative solution to a cooperative game: for any deviation of the set of elements in the stable set, there is a preferable element in the stable set. In spite of this appealing foundation of the stable set, three drawbacks appear: the stable set may be empty, the stable set may not be unique, and stable sets are difficult to compute.

In Gillies (1953) a new solution concept was introduced: the core. The core of the game is the set of efficient and coalitionally rational allocations. In other words, the core is the set of allocations that exactly share the value obtained by means of cooperation in such a way that all coalitions get at least the value that they can achieve by only cooperating among themselves. Although the core may be empty, it is one of the most accepted and studied solution concepts in cooperative game theory. It is well known that the core may be computed by means of a linear programming problem and that the core is internally stable although it may not be externally stable. Moreover, the class of cooperative games with a non-empty core is characterized as the class of balanced games (see Bondareva (1963) and Shapley (1967)), which definition only depends on the coalitional values of a game.

It is well-known that the core is contained in the stable set and that if the core is stable, then it is the only stable set (see Driessen (1988)). One of the open questions in cooperative game theory is the characterization of the class of games for which the core is stable. It is well-known that games with a large core (introduced in Sharkey (1982)) have a stable core. A game is said to have a large core if for any coalitional rational allocation (not necessarily efficient) there is a core element in which no player is better off than in the original allocation. The idea behind largeness of the core is that if only the value of the grand coalition is increased, then no player should be harmed by this, i.e. there is no core element in the new game that makes always some player worse off according to the core elements of the initial game.

Largeness of the core has been studied in the literature with respect to different properties and/or classes of games. Biswas, Parthasarathy and Ravindran (2001) analyzes largeness of the core with respect to stability of the core and provide a characterization of largeness of the core by means of extendability in the stronger sense. This condition establishes a relation between the elements of the lower bound of the upper core of every subgame and the elements of the core.

Biswas, Parthasarathy, Potters and Voorneveld (1999) analyzes largeness of the core for exact games. They show that exact games with at most four players have a large core, while for at least five players the result does not hold. Moreover, for totally balanced symmetric games, largeness of the core, stability of the core, and exactness are equivalent.

Van Gellekom, Potters and Reijnierse (1999) studies prosperity properties for cooperative games and 
show that largeness of the core is a prosperity property (i.e. we can always find a minimal value for the grand coalition for which the core is large). A characterization of largeness of the core is given by means of the extreme points of the upper core.

Azrieli and Lehrer (2007) provides a characterization of largeness of the core by means of the so called concavification of a game and the elements of the core of the game. Azrieli and Lehrer (2007) also provides an alternative proof to the equivalence of largeness of the core and strong extendability in the strongest sense by means of the concavification of a game.

In this paper we provide two new characterizations of largeness of the core that further analyze the structure of the core of this type of games. The first is given in terms of the basic solutions that provide extreme points of the core. The second is given by means of the set of bases needed to provide core and upper core elements.

All the characterizations of largeness of the core mentioned above are based on the structure of the core of the game (and/or subgames), or on the structure of the upper core of the game (and/or subgames), or in both. Although they provide an important insight into the structure of the core of games with a large core, these characterizations are difficult to apply in practice since many times the structure of the core and/or upper core are unknown.

Van Velzen, Hamers and Solymosi (2008) characterizes largeness of the core for chain-component additive games by means of minimal covering families of the set of players and corresponding inequalities. Van Velzen (2005) generalizes this result to the class of tree-component additive games. These characterizations do not depend on the structures of the core and/or upper core of the corresponding game, but only on the coalitional values of the game.

In this paper we generalize the result in van Velzen (2005) to the class of cooperative games. For this, we first show that the generalization of the condition in van Velzen (2005) implies non-emptiness of the core of a game. This step is not needed in van Velzen (2005) since tree-component additive games have always a non-empty core as shown in LeBreton, Owen and Weber (1992).

It is well known that largeness of the core and stability of the core are not equivalent in general. Van Gellekom et al. (1999) provides an example of a 6-players game with a stable core that is not large. By means of the new characterizations, we show that stability of the core and largeness of the core are equivalent for games with at most 5 players.

The structure of this paper is as follows. Section 2 recalls some basic game theoretic notions. Section 3 is devoted to the new characterizations of largeness of the core. Section 4 establishes the equivalence between stability and largeness of the core for games with at most 5 players. Section 5 recalls some results from the literature of largeness of the core and analyzes them by means of the new characterizations. Section 6 concludes with some final remarks. 


\section{Preliminaries}

A cooperative (transferable utility) game in characteristic function form is an ordered pair $(N, v)$ where $N$ is a finite set of $n=|N|$ players and $v: 2^{N} \rightarrow \mathbb{R}$ satisfies $v(\emptyset)=0$. In general, $v(S)$ represents the value of coalition $S$, i.e. the joint payoff that can be obtained by this coalition when its members decide to cooperate. A cooperative game can reflect rewards or costs. In this paper we study games on terms of rewards. The central question within a cooperative framework is how to share the revenues that are obtained by means of cooperation among the players, i.e. how to share the value of the grand coalition, $v(N)$, among the players. The imputation set of a game $(N, v), I(v)$, is the set of efficient allocations of $v(N)$ that are individually rational (i.e. to which no player can reasonably object). Formally, ${ }^{1}$

$$
\mathrm{I}(v)=\left\{x \in \mathbb{R}^{N} \mid x(N)=v(N), x_{i} \geq v(\{i\}) \text { for all } i \in N\right\}
$$

One of the most studied solution concepts in cooperative game theory is the core of a game, first introduced in Gillies (1953). The core of a game $(N, v)$, Core $(v)$, is the set of efficient allocations of $v(N)$ (i.e. exactly $v(N)$ is shared among the players) that are coalitionally rational (i.e. to which no coalition can reasonably object). Formally,

$$
\operatorname{Core}(v)=\left\{x \in \mathbb{R}^{N} \mid x(N)=v(N), x(S) \geq v(S) \text { for all } S \in 2^{N}\right\}
$$

It is well known that the core of a game is a polytope and therefore it can be expressed as the convex hull of its extreme points. The upper core of a game $(N, v), \mathrm{U}(v)$, is the set of not necessarily efficient allocations that are coalitionally rational. Formally,

$$
\mathrm{U}(v)=\left\{x \in \mathbb{R}^{N} \mid x(S) \geq v(S) \text { for all } S \in 2^{N}\right\}
$$

Given a game $(N, v)$ and $\alpha \in \mathbb{R}_{+}$, we define the game $\left(N, v^{\alpha}\right)$ by

$$
v^{\alpha}(S)= \begin{cases}v(S) & \text { if } S \in 2^{N} \backslash\{N\}, \\ v(N)+\alpha & \text { if } S=N\end{cases}
$$

Note that the upper core of $(N, v)$ can be written as the union of the cores of $\left(N, v^{\alpha}\right)$ with $\alpha \geq 0$, i.e. $\mathrm{U}(v)=\bigcup_{\alpha \in \mathbb{R}_{+}} \operatorname{Core}\left(v^{\alpha}\right)$.

Largeness of the core was first introduced in Sharkey (1982). The core of $(N, v)$ is said to be large if for every $x \in \mathrm{U}(v)$ there is a $y \in \operatorname{Core}(v)$ with $y_{i} \leq x_{i}$ for each $i \in N$. The lower bound set of $(N, v)$ was introduced in Sharkey (1982) and is defined as

$$
\mathrm{L}(v)=\{x \in U(v) \mid \text { there is no } y \in U(v) \text { such that } y \leq x \text { and } y \neq x\} .
$$

\footnotetext{
${ }^{1}$ Here and further, for any vector $x \in \mathbb{R}^{N}$ and any $S \subset N$, we denote $x(S):=\sum_{i \in S} x_{i}$.
} 
Note that $\operatorname{Core}(v) \subset \mathrm{L}(v)$ and $\mathrm{U}(v)=\mathrm{L}(v)+\mathbb{R}_{+}^{N}$. It follows immediately that the core of $(N, v)$ is large if and only if $\operatorname{Core}(v)=\mathrm{L}(v)$ (see Sharkey (1982) for a proof).

Since this paper focuses on largeness of the core, we assume without loss of generality that the game under consideration is nonnegative ${ }^{2}$.

Finally, given a finite set $N$ and $S \in 2^{\mathrm{N}} \backslash\{\emptyset\}$, the incidence vector of $S$ is defined as $e^{S} \in \mathbb{R}^{N}$ with $e_{i}^{S}=1$ if $i \in S$ and $e_{i}^{S}=0$ if $i \notin S$.

\section{New characterizations of largeness of the core}

In this section we provide three new characterizations of largeness of the core. For clarity of exposition all proofs and auxiliary lemmas are postponed to the appendix.

\subsection{Largeness of the core and minimal covers}

In this subsection we provide an alternative characterization of games with a large core based on minimal covers and corresponding minimal cover inequalities. Such a characterization was first proposed for the class of tree-component additive games in Van Velzen (2005). Here, we extend the result in Van Velzen (2005) to the general class of transferable utility games. For this, we first need to show that minimal cover inequalities imply non-emptiness of the core.

Given a finite set $N$, a collection $C \subset 2^{\mathrm{N}} \backslash\{\emptyset\}$ is called a minimal cover of $N$ if $C \neq\{N\}$ and the following two conditions are satisfied:

(CF1) $\cup_{S \in C} S=N$;

$(\mathrm{CF} 2) \cup_{S \in C \backslash\left\{S^{\prime}\right\}} S \neq N$ for all $S^{\prime} \in C$;

By condition (CF1), it follows that $C$ covers $N$; by condition (CF2), we have that no element of $C$ is superfluous. We denote by $\mathcal{M} \mathfrak{C}(N)$ the set of minimal covers of $N$.

Given a finite set $N$ and $C \in \mathcal{M} C(N)$, for every $i \in N$ we denote by $\xi(C, i)$ the number of times that $i$ is "extra covered" in $C$, i.e. $\xi(C, i)=|\{S \in C \mid i \in S\}|-1$. We denote by $E(C)$ the set of elements of $N$ that are" extra covered" by $C$, i.e. $E(C)=\{i \in N \mid \xi(C, i)>0\}$.

Given a minimal cover $C \in \mathcal{M C C}(N)$ of $N$, a vector $\mu \in \mathbb{R}_{+}^{2^{n}-1}$ is said to be a vector of minimal cover balanced weights (mc-balanced vector) for $C$ if

$$
\sum_{S \in 2^{\mathbb{N}} \backslash\{\emptyset\}} \mu_{S} e^{S}=\sum_{i \in N} \xi(C, i) e^{\{i\}}
$$

\footnotetext{
${ }^{2}$ Given a game $(N, v)$, we can always find a vector $a \in \mathbb{R}^{N}$ such that the game $(N, w)$ defined by $w(S)=v(S)+a(S)$ for every $S \in 2^{N}$ is nonnegative. It is well known that $\operatorname{Core}(w)=\{x+a \mid x \in \operatorname{Core}(v)\}$ and $\mathrm{U}(w)=\{x+a \mid x \in \mathrm{U}(v)\}$.
} 
Note that $\mu_{S}=0$ for every $S \not \subset E(C)$ by nonnegativity of $\mu$. We denote by $M(C)$ the set of mc-balanced vectors for $C$. It follows

$$
\sum_{S \in C} e^{S}=e^{N}+\sum_{S \in 2^{\mathrm{N}} \backslash\{\emptyset\}} \mu_{S} e^{S}
$$

for every $\mu \in M(C)$. Given a game $(N, v)$ and a minimal cover $C \in \mathcal{M} \mathcal{C}(N)$, the minimal cover inequality associated to $C$ is

$$
\sum_{S \in C} v(S) \leq v(N)+\max _{\mu \in M(C)}\left\{\sum_{S \in 2^{N} \backslash\{\emptyset\}} \mu_{S} v(S)\right\} .
$$

Next, we state the two main results of this subsection, which proofs can be found in the appendix.

Theorem 3.1. Let $(N, v)$ be a game satisfying all minimal cover inequalities. Then, $(N, v)$ has a non-empty core.

Theorem 3.2. A game $(N, v)$ has a large core if and only if all minimal cover inequalities are satisfied.

\subsection{Largeness of the core and basic solutions}

In this subsection we characterize largeness of the core in relation to the extreme points of the core. For this, we need to introduce some new notation.

Given a finite set $N$, a collection of coalitions $B=\left\{S_{1}, \ldots, S_{n}\right\}$ is called a base of $N$ if $\left\{e^{S_{1}}, \ldots, e^{S_{n}}\right\}$ is a base of $\mathbb{R}^{N}$. For every $R \in 2^{\mathrm{N}} \backslash\{\emptyset\}$, we denote by $\left\{\eta^{R}(S, B)\right\}_{S \in B}$ the collection of weights such that

$$
\sum_{S \in B} \eta^{R}(S, B) e^{S}=e^{R}
$$

If no confusion is to be expected, we omit $B$ from the notation. Note that this collection of weights is unique. We denote by $\mathbf{B}(N)$ the set of bases of $N$ and by $\mathbf{B}_{+}(N), \mathbf{B}_{-}(N)$, and $\mathbf{B}_{0}(N)$ the sets of bases with

$$
\begin{aligned}
& \mathbf{B}_{+}(N)=\left\{B \in \mathbf{B}(N) \mid N \in B \text { and } \eta^{\{i\}}(N) \geq 0 \text { for every } i \in N\right\}, \\
& \mathbf{B}_{-}(N)=\left\{B \in \mathbf{B}(N) \mid N \in B \text { and } \eta^{\{i\}}(N)<0 \text { for some } i \in N\right\}, \\
& \mathbf{B}_{0}(N)=\{B \in \mathbf{B}(N) \mid N \notin B\} .
\end{aligned}
$$

Note that $\left\{\mathbf{B}_{+}(N), \mathbf{B}_{-}(N), \mathbf{B}_{0}(N)\right\}$ forms a partition of $\mathbf{B}(N)$. Given a game $(N, v)$ and a base $B=$ $\left\{S_{1}, \ldots, S_{n}\right\} \in \mathbf{B}(N)$, we consider the system of linear equations

$$
x\left(S_{k}\right)=v\left(S_{k}\right) \text { for } \mathrm{k} \in\{1, \ldots, n\} .
$$

Since $B$ is a base of $N$, the system of equations has a unique solution which we denote by $x^{B}$. Analogously, given $\alpha>0$ we denote by $x^{B, \alpha}$ the unique solution to the system of linear equations given by

$$
x\left(S_{k}\right)=v^{\alpha}\left(S_{k}\right) \text { for } \mathrm{k} \in\{1, \ldots, n\} .
$$


The core and the upper core of a game can be computed as the set of optimal solutions to a series of systems of linear inequalities. Given a game $(N, v)$ and $\alpha \geq 0$, the systems of linear inequalities $\left(\mathrm{LI}^{\alpha}(v)\right)$ are defined as

$$
\begin{aligned}
\left(\mathrm{LI}^{\alpha}(v)\right) \quad- & \sum_{i \in N} x_{i} \geq-v(N)-\alpha \\
& \sum_{i \in N} x_{i} \geq v(N)+\alpha \\
& \sum_{i \in S} x_{i} \geq v(S) \quad \text { for every } S \in 2^{N} \backslash\{\emptyset, N\} .
\end{aligned}
$$

Then $\operatorname{Core}(v)=\operatorname{SOL}\left(\operatorname{LI}^{0}(v)\right)$ and $\mathrm{U}(v)=\bigcup_{\alpha \in \mathbb{R}_{+}} \operatorname{SOL}\left(\operatorname{LI}^{\alpha}(v)\right)$, where $\operatorname{SOL}\left(\operatorname{LI}^{\alpha}(v)\right)$ denotes the set of optimal solutions of $\operatorname{LI}^{\alpha}(v)$.

In order to characterize largeness of the core, we focus on necessary and sufficient conditions to guarantee that the core and the lower bound set coincide. The following result is a direct consequence of the fact that a vector $\bar{x}$ is an extreme point of $\operatorname{SOL}\left(\operatorname{LI}^{\alpha}(v)\right)$ if and only if the system $\operatorname{LI}^{\alpha}(v)$ evaluated in $\bar{x}$ contains at least $n$ tight equations that are linearly independent (see Chvàtal (1983)). The proof is therefore omitted.

Theorem 3.3. Let $(N, v)$ be a game. Then, ${ }^{3}$

$$
\operatorname{extr}(\operatorname{Core}(v))=\left\{x^{B} \mid B \in \mathbf{B}(N), x^{B} \in \operatorname{Core}(v)\right\}
$$

From now on, we denote by $\mathbf{B}(N, v)$ the set of bases of $N$ that provide extreme points of the core of $(N, v)$, i.e. $\mathbf{B}(N, v)=\left\{B \in \mathbf{B}(N) \mid x^{B} \in \operatorname{Core}(v)\right\}$. Analogously as above, we denote $\mathbf{B}_{+}(N, v), \mathbf{B}_{-}(N, v)$, and $\mathbf{B}_{0}(N, v)$.

The next example illustrates the type of core structure needed for a game to have a large core. This is done in terms of the extreme points of the core.

Example 3.1. Let $(N, v)$ be the game with $N=\{1,2,3\}$ and values $v(\{i\})=0$ for every $i \in N, v(\{1,2\})=$ $v(\{1,3\})=1, v(\{2,3\})=0$ and $v(N)=1.5$. In Figure 1 we represent the core of the game together with the core of $\left(N, v^{0.5}\right)$.
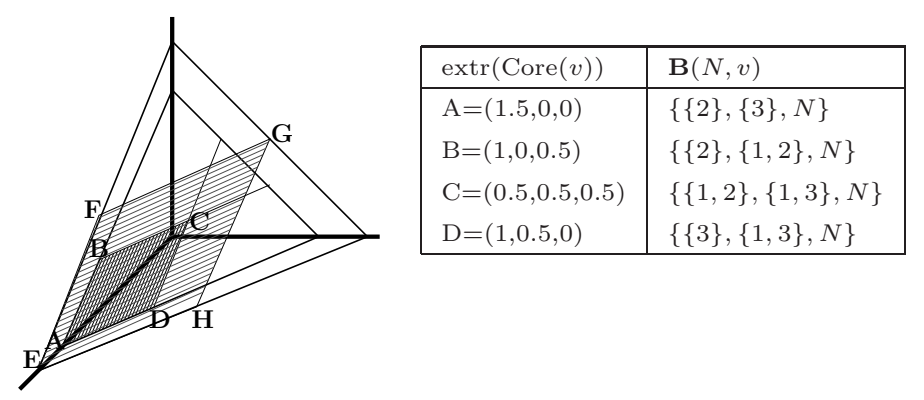

\begin{tabular}{|l|l|}
\hline $\operatorname{extr}\left(\operatorname{Core}\left(v^{0.5}\right)\right)$ & $\mathbf{B}\left(N, v^{0.5}\right)$ \\
\hline $\mathrm{E}=(2,0,0)$ & $\{\{2\},\{3\}, N\}$ \\
$\mathrm{F}=(1,0,1)$ & $\{\{2\},\{1,2\}, N\}$ \\
$\mathrm{G}=(0,1,1)$ & $\{\{1,2\},\{1,3\}, N\}$, \\
& $\{\{1\},\{1,2\}, N\}$, \\
& $\{\{1\},\{1,3\}, N\}$ \\
$\mathrm{H}=(1,1,0)$ & $\{\{3\},\{1,3\}, N\}$ \\
\hline
\end{tabular}

Figure 1: Core of $(N, v)$ and $\left(N, v^{0.5}\right)$ in Example 3.1.

\footnotetext{
${ }^{3}$ Here and further, extr denotes the set of "extreme points of".
} 
We have that

$$
\begin{aligned}
& \operatorname{Core}(v)=\operatorname{conv}\{(1.5,0,0),(1,0,0.5),(0.5,0.5,0.5),(1,0.5,0)\} \text { and } \\
& \mathbf{B}(N, v)=\{\{\{2\},\{3\}, N\},\{\{2\},\{1,2\}, N\},\{\{1,2\},\{1,3\}, N\},\{\{3\},\{1,3\}, N\}\} .
\end{aligned}
$$

Note that $\mathbf{B}(N, v)=\mathbf{B}_{+}(N, v) \cup \mathbf{B}_{-}(N, v)$ with $\mathbf{B}_{+}(N, v)=\{\{\{2\},\{3\}, N\},\{\{2\},\{1,2\}, N\},\{\{3\},\{1,3\}, N\}\}$ and $\left.\mathbf{B}_{-}(N, v)=\{\{1,2\},\{1,3\}, N\}\right\}$. It turns out that $(0.5,0.5,0.5)$ is obtained as the solution associated to $\{\{1,2\},\{1,3\}, N\}$ and satisfies:

$$
x^{B}(\{1\})=v(\{1,2\})+v(\{1,3\})-v(N), x^{B}(\{2\})=v(N)-v(\{1,3\}), x^{B}(\{3\})=v(N)-v(\{1,2\})
$$

and $x^{B}(\{1\})=0.5>0=v(\{1\})$. Therefore, if we increase the value of the grand coalition by $\alpha \in(0,0.5]$, the solution $x^{B, \alpha}$ is a core element of the new game $\left(N, v^{\alpha}\right)$ with

$$
x^{B, \alpha}(\{1\})=0.5-\alpha, x^{B, \alpha}(\{2\})=0.5+\alpha, x^{B, \alpha}(\{3\})=0.5+\alpha .
$$

It turns out that the game does not have a large core since for $\alpha \in(0,0.5], x^{B, \alpha} \in \mathrm{U}(v)$ and there is no $x \in \operatorname{Core}(v)$ with $x \leq x^{B, \alpha}$. This is related to the fact that if $x^{B, \alpha} \in \mathrm{U}(v)$ and there exists $x \in \operatorname{Core}(v)$ with $x \leq x^{B, \alpha}$, then $x=x^{B}$ (see Lemma A.3). It can be seen that $\left(N, v^{0.5}\right)$ has a large core.

Next, we provide the first characterization of largeness of the core. It describes the relation between the bases that provide core elements for the core of the game and for the core of the games resulting from the original by increasing the value of the grand coalition.

Theorem 3.4. A game $(N, v)$ has a large core if and only if $\mathbf{B}_{+}(N, v) \supset \mathbf{B}\left(N, v^{\alpha}\right)$ for every $\alpha>0$.

The following characterization of largeness of the core describes the type of extreme points of the core of a game with a large core.

Theorem 3.5. A game $(N, v)$ has a large core if and only if the following conditions are satisfied

(i) $\mathbf{B}_{+}(N, v)$ is non-empty;

(ii) if $B \in \mathbf{B}_{+}(N) \backslash \mathbf{B}_{+}(N, v)$, then there exists $R \in 2^{\mathrm{N}} \backslash\{\emptyset\}$ such that $\eta^{R}(N)=0$ and $x^{B}(R)<v(R)$.

(iii) if $B \in \mathbf{B}_{0}(N)$ with $x^{B}(N)>v(N)$, then there exists $R \in 2^{\mathrm{N}} \backslash\{\emptyset\}$ such that $x^{B}(R)<v(R)$.

(iv) if $B \in \mathbf{B}_{-}(N) \backslash \mathbf{B}_{-}(N, v)$, then at least one of the following three conditions is satisfied

(a) there exists $R \in 2^{\mathrm{N}} \backslash\{\emptyset\}$ such that $\eta^{R}(N)=0$ and $x^{B}(R)<v(R)$,

(b) there exists $R \in 2^{\mathrm{N}} \backslash\{\emptyset\}$ such that $\eta^{R}(N)<0$ and $x^{B}(R) \leq v(R)$, 
(c) $\min _{\substack{R \in 2^{N} \backslash\{\emptyset\} \\ \eta^{R}(N)<0}}\left\{\max \left\{0, \frac{1}{\left|\eta^{R}(N)\right|}\left(x^{B}(R)-v(R)\right)\right\}\right\}<\max _{\substack{R \in 2^{N} \backslash\{\emptyset\} \\ \eta^{R}(N)>0}}\left\{\frac{1}{\eta^{R}(N)}\left(v(R)-x^{B}(R)\right)\right\}$.

(v) if $B \in \mathbf{B}_{-}(N, v)$, then there exists $R \in 2^{\mathrm{N}} \backslash\{\emptyset\}$ such that $\eta^{R}(N)<0$ and $x^{B}(R)=v(R)$.

Condition (i) makes sure that $\mathbf{B}_{+}(N, v)$ is non-empty and therefore the core is non-empty. Condition (ii) makes sure that there are no bases in $\mathbf{B}_{+}(N)$ that provide upper core elements but do not provide a core element. Condition (iii) makes sure that there are no bases in $\mathbf{B}_{0}(N)$ that provide upper core elements not belonging to the core. Condition (iv) makes sure that there are no bases in $\mathbf{B}_{-}(N)$ that provide upper core elements but do not provide a core element. Condition (v) makes sure that there are no bases in $\mathbf{B}_{-}(N)$ that provide both core elements and upper core elements outside the core.

\section{Largeness of the core and stability}

An important open question in cooperative game theory is to determine whether the core of a game is stable in the von Neumann-Morgenstein sense. Although there is not a characterization of the class of games with a stable core, some classes of games have been analyzed for which the core is stable. It is well-known that a game with a large core has a stable set (see Theorem 3 in Sharkey (1982)). Moreover, it is known that a game with a stable core may not have a large core. In Van Gellekom et al. (1999) a 6 players game with a stable core that is not large is provided (see Example 1 in Section 4.2). The question is now what is the relation between largeness of the core and stability of the core for games with less than 6 players. Before answering this question, we recall the definition of stability of the core (á la von Neumann-Morgenstein).

Let $(N, v)$ be a game with a non-empty core. The core is called stable if for every imputation $x \in$ $\mathrm{I}(v) \backslash \operatorname{Core}(v)$ there exists a core element $y \in \operatorname{Core}(v)$ and a coalition $S \in 2^{\mathrm{N}} \backslash\{\emptyset\}$ with $x(S)<v(S)$ such that $y(S)=v(S)$ and $y_{i}>x_{i}$ for every $i \in S$.

It turns out that largeness of the core and stability of the core are equivalent for games with at most 5 players. The proof of this result is postponed to the appendix.

Theorem 4.1. A game $(N, v)$ with $|N| \leq 5$ has a stable core if and only if it has a large core.

\section{Largeness of the core in the literature}

\subsection{Necessary conditions for largeness of the core}

In Sharkey (1982) it is shown that subconvex games have a large core. A game $(N, v)$ is subconvex (see Sharkey (1982)) if for every partition $\mathbf{P}=\left\{P_{1}, \ldots, P_{k}\right\}$ of $\mathrm{N}$ and every collection of coalitions $\mathbf{Q}=\left\{Q_{1}, \ldots, Q_{k}\right\}$ 
such that $Q_{l} \subset \bigcup_{m=0}^{l-1} P_{m}$ and $P_{k} \cup Q_{k} \neq N$, where $P_{0}=\emptyset$, it follows

$$
\sum_{l=1}^{k}\left(v\left(P_{l} \cup Q_{l}\right)-v\left(Q_{l}\right)\right) \leq v(N) .
$$

An alternative proof to Sharkey's Theorem is by showing that every subconvex game satisfies the minimal cover inequalities.

Theorem 5.1 (Sharkey $(1982))$. If $(N, v)$ is subconvex, then $(N, v)$ satisfies all minimal cover inequalities.

Proof: Let $(N, v)$ be a subconvex game, we have to show that all minimal cover inequalities are satisfied. Let $C=\left\{S_{1}, \ldots, S_{k}\right\}$ be a minimal cover and define $P_{l}=S_{l} \backslash\left(\cup_{m=0}^{l-1} S_{m}\right)$ for $l \in\{1, \ldots, k\}$, where $S_{0}=\emptyset$, $Q_{1}=\emptyset$, and $Q_{l}=S_{l} \cap\left(\cup_{m=1}^{l-1} S_{m}\right)$ for $l \in\{2, \ldots, k\}$. Then,

$$
\begin{aligned}
\sum_{l=1}^{k} v\left(S_{l}\right) & =\sum_{l=1}^{k} v\left(P_{l} \cup Q_{l}\right)=\sum_{l=1}^{k}\left(v\left(P_{l} \cup Q_{l}\right)-v\left(Q_{l}\right)\right)+\sum_{l=1}^{k} v\left(Q_{l}\right) \\
& \leq v(N)+\sum_{l=1}^{k} v\left(Q_{l}\right) \leq v(N)+\max _{\mu \in M(C)}\left\{\sum_{S \in 2^{N} \backslash\{\emptyset\}} \mu_{S} v(S)\right\}
\end{aligned}
$$

where the first equality follows by definition of $\left\{P_{1} \ldots, P_{k}\right\}$ and $\left\{Q_{1} \ldots, Q_{k}\right\}$, the first inequality is a direct consequence of $(N, v)$ subconvex, and the last inequality is a consequence of $\tilde{\mu}$ defined as

$$
\tilde{\mu}_{S}= \begin{cases}\left|\left\{l \in\{1, \ldots, k\} \mid S=Q_{l}\right\}\right| & \text { if } S \in\left\{Q_{1} \ldots, Q_{k}\right\} \\ 0 & \text { otherwise }\end{cases}
$$

being an mc-balanced vector for $C$ by definition of $\left\{Q_{1} \ldots, Q_{k}\right\}$.

From the proof above, we see that the concept of subconvexity introduced in Sharkey (1982) is close to minimal cover inequalities. The only difference is that subconvexity explicitly selects the mc-balanced vector of weights to be $\mu_{Q_{l}}=1$ for $l \in\{2, \ldots, k\}$ and $\mu_{S}=0$ for $S \notin\left\{Q_{2}, \ldots, Q_{k}\right\}$.

Convex games where first introduced in Shapley (1971). Recall that a game $(N, v)$ is convex if $v(S)+$ $v(T) \leq v(S \cup T)+v(S \cap T)$ for every $S, T \in 2^{N}$. It readily follows that convex games are also subconvex and therefore have a large core. On page 180 in Sharkey (1982) it is stated "In fact it is easy to show that if $n=3$, then the core is large if and only if $(N, v)$ is convex". Note that Sharkey's remark is not true since a three players non superadditive game is not convex, but may have a large core. It turns out that subconvexity is equivalent to largeness of the core for games with at most three players.

Theorem 5.2. A game $(N, v)$ with $n \leq 3$ satisfies all minimal cover inequalities if and only if it is subconvex. Proof: If $N=\{1,2\}$, it easily follows that there is only one minimal cover: $\{\{1\},\{2\}\}$. Therefore, $(N, v)$ satisfies all minimal cover inequalities if and only if $(N, v)$ has a non-empty core, which for two players games is equivalent to both convexity and subconvexity. 
If $N=\{1,2,3\}$, then all minimal covers are either partitions of $N$ or of the form $\{\{i, j\},\{i, k\}\}$ with $\{i, j, k\}=N$. Let $\mathbf{P}=\left\{P_{1}, P_{2}, P_{3}\right\}$ with $P_{1}=\{i\}, P_{2}=\{j\}$, and $P_{3}=\{k\}$, then

$$
\sum_{l=1}^{3}\left(v\left(P_{l} \cup Q_{l}\right)-v\left(Q_{l}\right)\right)= \begin{cases}v(\{i\})+v(\{j\})+v(\{k\}) & \text { if } \mathbf{Q}=\{\emptyset, \emptyset, \emptyset\}, \\ v(\{j\})+v(\{i, k\}) & \text { if } \mathbf{Q}=\{\emptyset, \emptyset,\{i\}\}, \\ v(\{i\})+v(\{j, k\}) & \text { if } \mathbf{Q}=\{\emptyset, \emptyset,\{j\}\}, \\ v(\{i, j\})+v(\{i, k\})-v(\{i\}) & \text { if } \mathbf{Q}=\{\emptyset,\{i\},\{i\}\}, \\ v(\{i, j\})+v(\{j, k\})-v(\{j\}) & \text { if } \mathbf{Q}=\{\emptyset,\{i\},\{j\}\} .\end{cases}
$$

Let $\mathbf{P}=\left\{P_{1}, P_{2}\right\}$. If $P_{1}=\{i, j\}$ and $P_{2}=\{k\}$, then

$$
\sum_{l=1}^{2}\left(v\left(P_{l} \cup Q_{l}\right)-v\left(Q_{l}\right)\right)= \begin{cases}v(\{i, j\})+v(\{k\}) & \text { if } \mathbf{Q}=\{\emptyset, \emptyset\}, \\ v(\{i, j\})+v(\{i, k\})-v(\{i\}) & \text { if } \mathbf{Q}=\{\emptyset,\{i\}\}, \\ v(\{i, j\})+v(\{j, k\})-v(\{j\}) & \text { if } \mathbf{Q}=\{\emptyset,\{j\}\} .\end{cases}
$$

If $P_{1}=\{k\}$ and $P_{2}=\{i, j\}$, then $\mathbf{Q}=\{\emptyset, \emptyset\}$ and

$$
\sum_{l=1}^{2}\left(v\left(P_{l} \cup Q_{l}\right)-v\left(Q_{l}\right)\right)=v(\{i, j\})+v(\{k\}) .
$$

Therefore, it is easily seen that all minimal cover inequalities are satisfied if and only if the game is subconvex.

The following example illustrates that subconvexity and largeness of the core are not equivalent for games with more than three players.

Example 5.1. Let $(N, v)$ be the game with $N=\{1,2,3,4\}$ and values given in Table 1 .

\begin{tabular}{|l|c|c|c|c|c|c|c|c|c|c|c|c|c|c|c|}
\hline$S$ & $\{1\}$ & $\{2\}$ & $\{3\}$ & $\{4\}$ & $\{1,2\}$ & $\{1,3\}$ & $\{1,4\}$ & $\{2,3\}$ & $\{2,4\}$ & $\{3,4\}$ & $\{1,2,3\}$ & $\{1,2,4\}$ & $\{1,3,4\}$ & $\{2,3,4\}$ & $N$ \\
\hline$v(S)$ & 5 & 5 & 0 & 0 & 5 & 5 & 5 & 5 & 5 & 0 & 10 & 10 & 5 & 5 & 14 \\
\hline
\end{tabular}

Table 1: Coalitional values of the game in Example 5.1.

It is readily seen that all minimal cover inequalities are satisfied since $v(S) \leq \sum_{i \in S} v(\{i\})$ for every $S \in 2^{\mathrm{N}} \backslash\{\emptyset\}$ with $1<|S|<4$, and $\sum_{i \in N} v(\{i\})<v(N)$. Note that the game is not subconvex by taking $\mathbf{P}=\{\{1,2,3\},\{4\}\}$ and $\mathbf{Q}=\{\{\emptyset\},\{1,2\}\}$ since

$$
v(\{1,2,3\})+(v(\{1,2,4\}-v(\{1,2\})))=10+(10-5)=15 \not \leq 14=v(N) .
$$

\subsection{Largeness of the core and exactness}

In Biswas et al. (1999) the relation between largeness of the core and exactness is stablished. A game $(N, v)$ is exact (Schmeidler (1972)) if for every coalition $R \in 2^{\mathrm{N}} \backslash\{\emptyset\}$ there is a core allocation $x \in$ Core $(v)$ with 
$x(R)=v(R)$.

Csóka, Herings and Kóczy (2007) provides a characterization of exact games in terms of balanced conditions. A collection $E \subset 2^{\mathrm{N}} \backslash\{\emptyset\}$ is exactly balanced if there exist a vector $\varepsilon \in \mathbb{R}^{2^{n}-1}$ such that $\varepsilon_{T} \in \mathbb{R}$ for some $T \in E, \varepsilon_{S}>0$ for $S \in E, S \neq T$, and $\sum_{R \in E} \varepsilon_{R} e^{R}=e^{N}$. A game $(N, v)$ is exactly balanced if $\sum_{R \in E} \varepsilon_{R} v(R) \leq v(N)$ for all exactly balanced collections. It follows (see Csóka et al. (2007)) that a game is exact if and only if it is exactly balanced.

In Biswas et al. (1999) it is shown that every exact game with at most four players has a large core. The proof of this result can now be simplified by using Theorem 3.2 and the characterization of exact games by means of exactly balanced collections.

Theorem 5.3 (Biswas et al. (1999)). If $(N, v)$ is an exact game and $n \leq 4$, then $(N, v)$ satisfies all minimal cover inequalities. If $n \geq 5$, there are exact games $(N, v)$ not satisfying all minimal cover inequalities.

Proof: Let $(N, v)$ be an exact game. If $n=2$, it follows that exactness of the game is equivalent to nonemptiness of the core, which is equivalent to all minimal cover inequalities being satisfied (see proof of Theorem 5.1).

If $n=3$, it can be seen that all minimal covers are either a partition of $N$, or of the following form: $\{\{i, j\},\{i, k\}\}$ with $\{i, j, k\}=N$. It is readily seen that a partition is an exactly balanced collection and therefore the minimal cover inequalities for partitions of $N$ are satisfied. We have to show that the minimal cover inequalities are satisfied for $\{\{i, j\},\{i, k\}\}$. Note that the collection $\{\{i\},\{i, j\},\{i, k\}\}$ is exactly balanced, with $\varepsilon_{\{i\}}=-1$ and $\varepsilon_{\{i, j\}}=\varepsilon_{\{i, k\}}=1$. Therefore

$$
\begin{aligned}
v(\{i, j\})+v(\{i, k\}) & =-v(\{i\})+v(\{i, j\})+v(\{i, k\})+v(\{i\}) \\
& \leq v(N)+v(\{i\}) \\
& \leq v(N)+\max _{\mu \in M(C)}\left\{\sum_{S \in 2^{\mathbb{N}} \backslash\{\emptyset\}} \mu_{S} v(S)\right\}
\end{aligned}
$$

where the first inequality follows because the game is exact and therefore exactly balance, and the last inequality follows since the vector which assigns 1 to coalition $\{i\}$ and 0 to the other coalitions is mcbalanced for $C$.

If $n=4$, it can be seen that all minimal covers are either a partition of $N$, or of the following form: $\{\{j\},\{i, k\},\{i, l\}\},\{\{i, j\},\{i, k\},\{i, l\}\},\{\{i, j\},\{i, k, l\}\}$ and $\{\{i, j, k\},\{i, j, l\}\}$, with $\{i, j, k, l\}=N$. Since every partition of $N$ is exactly balanced, we only have to show that the minimal cover inequalities are satisfied for the four above-mentioned types.

Note that $\{\{j\},\{i, k\},\{i, l\}\}$ can be transformed in the exactly balanced collection $\{\{i\},\{j\},\{i, k\},\{i, l\}\}$ with exactly balanced vector $\varepsilon$ given by $\varepsilon_{\{i\}}=-1, \varepsilon_{\{j\}}=\varepsilon_{\{i, j\}}=\varepsilon_{\{i, k\}}=1$. Analogously, $\{\{i, j\},\{i, k\},\{i, l\}\}$ 
can be transformed in the exactly balanced collection $\{\{i\},\{i, j\},\{i, k\},\{i, l\}\}$ with exactly balanced vector $\varepsilon$ given by $\varepsilon_{\{i\}}=-2, \varepsilon_{\{i, j\}}=\varepsilon_{\{i, k\}}=\varepsilon_{\{i, l\}}=1 ;\{\{i, j\},\{i, k, l\}\}$ can be transformed in the exactly balanced collection $\{\{i\},\{i, j\},\{i, k, l\}\}$ with exactly balanced vector $\varepsilon$ given by $\varepsilon_{\{i\}}=-1, \varepsilon_{\{i, j\}}=\varepsilon_{\{i, k, l\}}=1$; $\{\{i, j, k\},\{i, j, l\}\}$ can be transformed in the exactly balanced collection $\{\{i, j\},\{i, j, k\},\{i, j, l\}\}$ with exactly balanced vector $\varepsilon$ given by $\varepsilon_{\{i, j\}}=-1, \varepsilon_{\{i, j, k\}}=\varepsilon_{\{i, j, l\}}=1$. Following the same lines as in the case $n=3$, it is readily seen that the exactly balanced conditions of the derived exactly balanced collections imply the minimal cover inequalities.

If $n \geq 5$, it easily follows that the minimal cover $\{\{i, j, k\},\{i, j, l\},\{i, m\}\}$, where $\{i, j, k, l, m\}=N$, cannot be transformed in an exactly balanced collection. Therefore, exactness does not need to imply all minimal cover inequalities.

\section{$6 \quad$ Final remarks}

In Theorem 3.5 we have characterized largeness of the core in terms of the extreme solutions, $x^{B}$, to a system of equations. Note that the result could be written down in terms of weights and coalitional values following Lemma A.2 (iii), which would only difficult readability. The main contribution of Theorem 3.5 is to understand the structure of the core of a game with a large core. According to Theorem 3.4, the set of bases that provide core elements for $(N, v)$ either remains the same, or decreases when we consider $\left(N, v^{\alpha}\right)$ with $\alpha>0$. In this sense, the "core is large" since the number of bases needed to describe the core of a game is non increasing on the value of the grand coalition.

Note that both the core and the upper core of a game $(N, v)$ may be described by means of the essential coalitions of the core. A coalition $S \in 2^{\mathrm{N}} \backslash\{\emptyset\}$ is called essential if $v(S)>\sum_{l=1}^{t} v\left(S^{l}\right)$ for any non-trivial partition $\left\{S^{1}, \ldots, S^{t}\right\}$ of $S$. Hence, we can restrict Theorems 3.1 and 3.2 to the set of minimal covers which elements are essential coalitions of the game. Analogously, we can restrict Theorem 3.4 and Theorem 3.5 to the set of bases which elements are essential coalitions of the game. This may be interesting when applying the results to a specific game in order to reduce computational time.

\section{Appendix}

\section{Largeness of the core and minimal covers}

In this part of the appendix, we show the two main results in Subsection 3.1. For this, we first provide an auxiliary lemma. 
Lemma A.1. Let $(N, v)$ be a game and let $C \in \mathcal{M} \mathfrak{C}(N)$. Then it follows

(i) $\max _{\mu \in M(C)}\left\{\sum_{S \in 2^{N} \backslash\{\emptyset\}} \mu_{S} v(S)\right\}=\min \left\{\sum_{i \in E(C)} \xi(C, i) x_{i} \mid x(S) \geq v(S)\right.$ for every $\left.S \subset E(C)\right\}$;

(ii) for every $\bar{\mu} \in \arg \max _{\mu \in M(C)}\left\{\sum_{S \in 2^{N} \backslash\{\emptyset\}} \mu_{S} v(S)\right\}$, there exists $x \in \mathbb{R}^{E(C)}$ such that $x(S) \geq v(S)$ for every $S \subset E(C), x(S)=v(S)$ for every $S \subset E(C)$ with $\bar{\mu}_{S}>0$, and $\sum_{i \in E(C)} \xi(C, i) x_{i}=$ $\max _{\mu \in M(C)}\left\{\sum_{S \in 2^{N} \backslash\{\emptyset\}} \mu_{S} v(S)\right\}$.

Proof: Note that equation (3.3) can be written as the following linear programming problem:

$$
\begin{aligned}
& \max \sum_{S \in 2^{\mathbb{N}} \backslash\{\emptyset\}} \mu_{S} v(S) \\
& \text { st } \\
& \sum_{S \in 2^{\mathbb{N}} \backslash\{\emptyset\}: S \ni i} \mu_{S} \leq \xi(C, i) \quad \text { for every } i \in N, \\
& \mu_{S} \geq 0 \quad \text { for every } S \in 2^{\mathrm{N}} \backslash\{\emptyset\} .
\end{aligned}
$$

Due to nonnegativity of $\mu$, it can be alternatively written as the linear programming problem:

$$
\begin{aligned}
& \max \sum_{S \in 2^{E(C)} \backslash\{\emptyset\}} \mu_{S} v(S) \\
& \text { st } \\
& \sum_{S \in 2^{E(C)} \backslash\{\emptyset\}: S \ni i} \mu_{S} \leq \xi(C, i) \quad \text { for every } i \in E(C), \\
& \mu_{S} \geq 0 \quad \text { for every } S \in 2^{E(C)} \backslash\{\emptyset\} \text {, }
\end{aligned}
$$

which dual problem is

$$
\begin{aligned}
& \min _{i \in E(C)} \xi(C, i) x_{i} \\
& \text { st } \\
& \sum_{i \in S} x_{i} \geq v(S) \quad \text { for every } S \in 2^{E(C)} \backslash\{\emptyset\} .
\end{aligned}
$$

(Note that the constraint $x_{i} \geq 0$ for every $i \in E(C)$ has been omitted by nonnegativity of $v$ ).

(i) immediately follows by the Strong Duality Theorem (see Dantzig and Thapa (2003)).

(ii) immediately follows by the Complementary Slackness Theorem (see Dantzig and Thapa (2003)).

Proof of Theorem 3.1. We construct an allocation vector $x \in \mathbb{R}^{N}$ and show that $x \in \operatorname{Core}(v)$. Let $C=\left\{S_{1}, \ldots, S_{k}\right\} \in \mathcal{M} \mathcal{C}(N)$ be such that

$$
\sum_{l=1}^{k} v\left(S_{l}\right)-\max _{\mu \in M(C)}\left\{\sum_{R \in 2^{N} \backslash\{\emptyset\}} \mu_{R} v(R)\right\} \geq \sum_{R \in \tilde{C}} v(R)-\max _{\mu \in M(\tilde{C})}\left\{\sum_{R \in 2^{N} \backslash\{\emptyset\}} \mu_{R} v(R)\right\} \text { for every } \tilde{C} \in \mathcal{M C}(N)
$$


Without loss of generality, let

$$
\begin{aligned}
S_{1} \backslash E(C) & =\left\{1,2, \ldots, i_{1}\right\}, \\
S_{2} \backslash E(C) & =\left\{i_{1}+1, i_{1}+2, \ldots, i_{2}\right\}, \\
& \vdots \\
S_{k} \backslash E(C) & =\left\{i_{k-1}+1, i_{k-1}+2, \ldots, i_{k}\right\}, \\
E(C) & =\left\{i_{k}+1, i_{k}+2, \ldots, n\right\} .
\end{aligned}
$$

By Lemma A.1 (ii), there exists $\bar{x} \in \mathbb{R}^{E(C)}$ such that

$$
\bar{x}(R) \geq v(R) \text { for every } R \in 2^{E(C)} \backslash\{\emptyset\}
$$

and

$$
\sum_{i \in E(C)} \xi(C, i) \bar{x}_{i}=\max _{\mu \in M(C)}\left\{\sum_{R \in 2^{\mathbb{N}} \backslash\{\emptyset\}} \mu_{R} v(R)\right\} .
$$

Define $x \in \mathbb{R}^{N}$ as follows: $x_{i}=\bar{x}_{i}$ for every $i \in E(C)$ and $x_{i}$ is recursively defined for every $i \in N \backslash E(C)$ in the following way:

$$
\begin{array}{ll}
x_{1}=\max _{R \subset E(C)}\{v(R \cup\{1\})-x(R)\} & \text { if } i_{1} \neq 1, \\
x_{i}=\max _{R \subset E(C) \cup\{1, \ldots, i-1\}}\{v(R \cup\{i\})-x(R)\} & \text { if } i \in N \backslash E(C) \text { and } i \notin\left\{1, i_{1}, \ldots, i_{k}\right\}, \\
\left.x_{i_{l}}=v\left(S_{l}\right)-x\left(S_{l} \backslash\left\{i_{l}\right\}\right)\right\} & \text { if } l \in\{1, \ldots, k-1\}, \\
x_{i_{k}}=v(N)-x\left(N \backslash\left\{i_{k}\right\}\right) . &
\end{array}
$$

For every $i \in\left\{1, \ldots, i_{k}\right\} \backslash\left\{i_{1}, i_{2}, \ldots, i_{k}\right\}$, fix $T^{i} \subset\{1,2, \ldots, i-1\} \cup E(C)$ such that

$$
x_{i}=v\left(T^{i} \cup\{i\}\right)-x\left(T^{i}\right) .
$$

Next, we show that $x \in \operatorname{Core}(v)$. Efficiency immediately follows from the definition of $x_{i_{k}}$ (see equation (A.7)). We recursively show coalitional rationality. Let $S_{0}=E(C)$. If $S \subset S_{0}$, then it readily follows that $x(S) \geq v(S)$ by definition of $x$ and equation (A.2). Next, assume that $x(S) \geq v(S)$ for every $S \subset \cup_{l=0}^{t-1} S_{l}$ with $t \leq k$. Let $S \subset \cup_{l=1}^{t} S_{l}$ such that $S \cap\left(S_{t} \backslash S_{0}\right) \neq \emptyset$. We distinguish between two cases: $i_{t} \notin S$ and $i_{t} \in S$.

\section{Case 1: $i_{t} \notin S$.}

In this case, let $i \in S_{t}$ be such that $S \subset\left(\cup_{l=0}^{t-1} S_{l}\right) \cup\left\{i_{t-1}+1, \ldots, i\right\}$. Note that $i \neq i_{t}$ by assumption. It follows that

$$
x(S)=x_{i}+x(S \backslash\{i\}) \geq v(S)-x(S \backslash\{i\})+x(S \backslash\{i\})=v(S)
$$

where the inequality is an immediate consequence of the definition of $x_{i}$ (see equation (A.5)). 


\section{Case 2: $i_{t} \in S$.}

For this case we construct a minimal cover $\tilde{C}$ based on $C$ where coalition $S_{t}$ is substituted by $S$ and coalitions $T^{j_{t_{1}}^{1}} \cup\left\{j_{t_{1}}^{1}\right\}, \ldots, T^{j_{t_{r}}^{r}} \cup\left\{j_{t_{r}}^{r}\right\}$, with $j_{t_{1}}^{1}, \ldots, j_{t_{r}}^{r} \in S_{t} \backslash\left(S \cup S_{0}\right)$, as follows. If $S_{t} \backslash S_{0} \subset S$, define $\tilde{C}_{0}=\left\{S_{1}, \ldots, S_{t-1}, S, S_{t+1}, \ldots, S_{k}\right\}$. Otherwise, let $\left\{j_{1}^{1}, \ldots, j_{t_{1}}^{1}\right\}=S_{t} \backslash\left(S \cup S_{0}\right)$ with $j_{1}^{1}<\ldots<j_{t_{1}}^{1}$. If $\left\{j_{1}^{1}, \ldots, j_{t_{1}}^{1}\right\} \subset T^{j_{t_{1}}^{1}} \cup\left\{j_{t_{1}}^{1}\right\}$, define $\tilde{C}_{0}=\left\{S_{1}, \ldots, S_{t-1}, S, T^{j_{t_{1}}^{1}} \cup\left\{j_{t_{1}}^{1}\right\}, S_{t+1}, \ldots, S_{k}\right\}$. Otherwise, define $\left\{j_{1}^{2}, \ldots, j_{t_{2}}^{2}\right\}=\left\{j_{1}^{1}, \ldots, j_{t_{1}}^{1}\right\} \backslash\left(T^{j_{t_{1}}^{1}} \cup\left\{j_{t_{1}}^{1}\right\}\right)$ and continue with this procedure. After $r\left(\leq t_{1}\right)$ steps we obtain $\tilde{C}_{0}=\left\{S_{1}, \ldots, S_{t-1}, S, T^{j_{t_{1}}^{1}} \cup\left\{j_{t_{1}}^{1}\right\}, \ldots, T^{j_{t_{r}}^{r}} \cup\left\{j_{t_{r}}^{r}\right\}, S_{t+1}, \ldots, S_{k}\right\}$. It readily follows that $N=\cup_{R \in \tilde{C}_{0}} R$ since $S_{t} \backslash\left(S \cup S_{0}\right) \subset \cup_{l=1}^{r}\left(T^{j_{t_{l}}^{l}} \cup\left\{j_{t_{l}}^{l}\right\}\right)$ by construction of $\tilde{C}_{0}$. Moreover, $i_{t}>j_{t_{1}}^{1}>\ldots>j_{t_{r}}^{r}$ and therefore $i_{t} \notin T^{j_{t_{l}}^{l}}$ for every $l \in\{1, \ldots, r\}$ and $j_{l^{\prime}}^{l^{\prime}} \notin T^{j_{t_{l}}^{l}}$ for every $l \in\{1, \ldots, r-1\}$ and $l^{\prime} \in\{l+1, \ldots, r\}$ by definition of $T^{j_{t_{l}}^{l}}$. If $\tilde{C}_{0} \in \mathcal{M C}(N)$, then $\tilde{C}=\tilde{C}_{0}$. If $\tilde{C}_{0}$ is no minimal cover, then we have that,

$$
\begin{aligned}
& \left|\left\{R \in \tilde{C}_{0} \mid i_{t} \in R\right\}\right|=|\{S\}|=1, \\
& \left|\left\{R \in \tilde{C}_{0} \mid j_{t_{l}}^{l} \in R\right\}\right|=\left|\left\{T^{j_{t_{l}}^{l}} \cup\left\{j_{t_{l}}^{l}\right\}\right\}\right|=1 \text { for every } l \in\{1, \ldots, r\},
\end{aligned}
$$

and

$$
\left|\left\{R \in \tilde{C}_{0} \mid i_{l} \in R\right\}\right|=\left|\left\{S_{l}\right\}\right|=1 \text { for every } l \in\{t+1, \ldots, k\}
$$

by construction of $\tilde{C}_{0}$. Therefore, there exist $I \subset\{1, \ldots, t-1\}$ such that $\tilde{C}=\tilde{C}_{0} \backslash\left\{S_{l} \mid l \in I\right\} \in \mathcal{M C}(N)$. It readily follows that

$$
\xi(\tilde{C}, i)=\xi(C, i)-\left|\left\{l \in I \cup\{t\} \mid i \in S_{l}\right\}\right|+\left|\left\{R \in\left\{S, T^{j_{t_{1}}^{1}} \cup\left\{j_{t_{1}}^{1}\right\}, \ldots, T^{j_{t_{r}}^{r}} \cup\left\{j_{t_{r}}^{r}\right\}\right\} \mid i \in R\right\}\right|
$$

for every $i \in N$ and as a consequence we have that

$$
E(\tilde{C}) \subset E(C) \cup\left\{1, \ldots, i_{t}-1\right\}=\left(\cup_{l=0}^{t} S_{l}\right) \backslash\left\{i_{t}\right\}
$$

since $T^{j_{t_{l}}^{l}} \subset E(C) \cup\left\{1,2, \ldots, j_{t_{l}}^{l}-1\right\}$ for every $l \in\{1, \ldots, r\}$. Next, we have to distinguish between two subcases: $t<k$ and $t=k$.

Subcase 2.1: $t<k$.

In this subcase it follows $x(S)=x_{i_{t}}+x\left(S \backslash\left\{i_{t}\right\}\right)=v\left(S_{t}\right)-x\left(S_{t} \backslash\left\{i_{t}\right\}\right)+x\left(S \backslash\left\{i_{t}\right\}\right)$, where the second equality follows by definition of $x_{i_{t}}$ (see equation (A.6)). Therefore we have to show that

$$
v(S) \leq v\left(S_{t}\right)-x\left(S_{t} \backslash\left\{i_{t}\right\}\right)+x\left(S \backslash\left\{i_{t}\right\}\right) .
$$

Note that

$$
\begin{aligned}
& v(S)+\sum_{l=1}^{r} v\left(T^{j_{t_{l}}^{l}} \cup\left\{j_{t_{l}}^{l}\right\}\right)+\sum_{l=1}^{t-1} v\left(S_{l}\right)-\sum_{l \in I} x\left(S_{l}\right)+\sum_{l=t+1}^{k} v\left(S_{l}\right)-\max _{\mu \in M(\tilde{C})}\left\{\sum_{R \in 2^{N} \backslash\{\emptyset\}} \mu_{R} v(R)\right\}= \\
& v(S)+\sum_{l=1}^{r} v\left(T^{j_{t_{l}}^{l}} \cup\left\{j_{t_{l}}^{l}\right\}\right)+\sum_{\substack{l=1 \\
l \notin I}}^{t-1} v\left(S_{l}\right)+\sum_{l=t+1}^{k} v\left(S_{l}\right)-\max _{\mu \in M(\tilde{C})}\left\{\sum_{R \in 2^{N} \backslash\{\emptyset\}} \mu_{R} v(R)\right\}=
\end{aligned}
$$




$$
\begin{aligned}
& \sum_{R \in \tilde{C}} v(R)-\max _{\mu \in M(\tilde{C})}\left\{\sum_{R \in 2^{N} \backslash\{\emptyset\}} \mu_{R} v(R)\right\} \leq \\
& \sum_{l=1}^{k} v\left(S_{l}\right)-\max _{\mu \in M(C)}\left\{\sum_{R \in 2^{N} \backslash\{\emptyset\}} \mu_{R} v(R)\right\}= \\
& \sum_{l=1}^{k} v\left(S_{l}\right)-\sum_{i \in N} \xi(C, i) x_{i}
\end{aligned}
$$

where the first equality follows because $v\left(S_{l}\right)=x\left(S_{l}\right)$ for every $l \in\{1, \ldots, k-1\}$ by definition of $x_{i_{l}}$ (see equation (A.6)), the inequality is a direct consequence of equation (A.1), and the last equality follows because $\xi(C, i)=0$ for $i \notin E(C)$, together with the definition of $x$ and equation (A.3). Hence,

$$
\begin{aligned}
v(S) \leq & v\left(S_{t}\right)-\sum_{l=1}^{r} v\left(T^{j_{t_{l}}^{l}} \cup\left\{j_{t_{l}}^{l}\right\}\right)+\sum_{l \in I} x\left(S_{l}\right)+\max _{\mu \in M(\tilde{C})}\left\{\sum_{R \in 2^{N} \backslash\{\emptyset\}} \mu_{R} v(R)\right\}-\sum_{i \in N} \xi(C, i) x_{i} \\
\leq & v\left(S_{t}\right)-\sum_{l=1}^{r} v\left(T^{j_{t_{l}}^{l}} \cup\left\{j_{t_{l}}^{l}\right\}\right)+\sum_{l \in I} x\left(S_{l}\right)+\max _{\mu \in M(\tilde{C})}\left\{\sum_{R \in 2^{N} \backslash\{\emptyset\}} \mu_{R} x(R)\right\}-\sum_{i \in N} \xi(C, i) x_{i} \\
= & v\left(S_{t}\right)-\sum_{l=1}^{r} x\left(T^{j_{t_{l}}^{l}} \cup\left\{j_{t_{l}}^{l}\right\}\right)+\sum_{l \in I} x\left(S_{l}\right)+\sum_{i \in N} \xi(\tilde{C}, i) x_{i}-\sum_{i \in N} \xi(C, i) x_{i} \\
= & v\left(S_{t}\right)-\sum_{l=1}^{r} x\left(T^{j_{t_{l}}^{l}} \cup\left\{j_{t_{l}}^{l}\right\}\right)+\sum_{l \in I} x\left(S_{l}\right)+\left[x(S)+\sum_{l=1}^{r} x\left(T^{j_{t_{l}}^{l}} \cup\left\{j_{t_{l}}^{l}\right\}\right)+\sum_{l=1}^{t-1} x\left(S_{l}\right)+\sum_{l=t+1}^{k} x\left(S_{l}\right)-x(N)\right] \\
& -\left[\sum_{l=1}^{k} x\left(S_{l}\right)-x(N)\right] \\
= & v\left(S_{t}\right)-x\left(S_{t}\right)+x(S) \\
= & v\left(S_{t}\right)-x\left(S_{t} \backslash\left\{i_{t}\right\}\right)+x\left(S \backslash\left\{i_{t}\right\}\right)
\end{aligned}
$$

where the first inequality follows by reordering the terms in the inequality derived above; the second inequality follows by equation(A.9) and $v(R) \leq x(R)$ for every $R \in\left(\cup_{l=0}^{t} S_{l}\right) \backslash\left\{i_{t}\right\}$ by induction and Case 1 ; the first equality follows by both the definition of $x_{j_{t_{l}}^{l}}$ (see equation (A.5)) and $T^{j_{t_{l}}^{l}}$ (see equation (A.8)), and the definition of mc-balanced vector for $\tilde{C}$; the second equality is a direct consequence of equations (3.1) and (3.2); the last equality readily follows since $i_{t} \in S \cap S_{t}$.

Subcase 2.2: $t=k$.

In this subcase it follows $x(S)=x_{i_{k}}+x\left(S \backslash\left\{i_{k}\right\}\right)=v(N)-x\left(N \backslash\left\{i_{k}\right\}\right)+x\left(S \backslash\left\{i_{k}\right\}\right)$. Therefore we have to show that

$$
v(S) \leq v(N)-x\left(N \backslash\left\{i_{k}\right\}\right)+x\left(S \backslash\left\{i_{k}\right\}\right)
$$


Note that

$$
\sum_{\substack{l=1 \\ l \notin I}}^{k-1} v\left(S_{l}\right)+v(S)+\sum_{l=1}^{r} v\left(T^{j_{t_{l}}^{l}} \cup\left\{j_{t_{l}}^{l}\right\}\right)=\sum_{R \in \tilde{C}} v(R) \leq v(N)+\max _{\mu \in M(\tilde{C})}\left\{\sum_{R \in 2^{N} \backslash\{\emptyset\}} \mu_{R} v(R)\right\}
$$

since $(N, v)$ satisfies all minimal cover inequalities. Hence,

$$
\begin{aligned}
& v(S) \leq v(N)-\sum_{\substack{l=1 \\
l \notin I}}^{k-1} v\left(S_{l}\right)-\sum_{l=1}^{r} v\left(T^{j_{t_{l}}^{l}} \cup\left\{j_{t_{l}}^{l}\right\}\right)+\max _{\mu \in M(\tilde{C})}\left\{\sum_{R \in 2^{N} \backslash\{\emptyset\}} \mu_{R} v(R)\right\} \\
& =v(N)-\sum_{l=1}^{k-1} v\left(S_{l}\right)+\sum_{l \in I} x\left(S_{l}\right)-\sum_{l=1}^{r} v\left(T^{j_{t_{l}}^{l}} \cup\left\{j_{t_{l}}^{l}\right\}\right)+\max _{\mu \in M(\tilde{C})}\left\{\sum_{R \in 2^{N} \backslash\{\emptyset\}} \mu_{R} v(R)\right\} \\
& \leq v(N)-\sum_{l=1}^{k-1} v\left(S_{l}\right)+\sum_{l \in I} x\left(S_{l}\right)-\sum_{l=1}^{r} v\left(T^{j_{t_{l}}^{l}} \cup\left\{j_{t_{l}}^{l}\right\}\right)+\max _{\mu \in M(\tilde{C})}\left\{\sum_{R \in 2^{N} \backslash\{\emptyset\}} \mu_{R} x(R)\right\} \\
& =v(N)-\sum_{l=1}^{k-1} x\left(S_{l}\right)+\sum_{l \in I} x\left(S_{l}\right)-\sum_{l=1}^{r} x\left(T^{j_{t_{l}}^{l}} \cup\left\{j_{t_{l}}^{l}\right\}\right)+\sum_{i \in N} \xi(\tilde{C}, i) x_{i} \\
& =v(N)-\sum_{l=1}^{k-1} x\left(S_{l}\right)+\sum_{l \in I} x\left(S_{l}\right)-\sum_{l=1}^{r} x\left(T^{j_{t_{l}}^{l}} \cup\left\{j_{t_{l}}^{l}\right\}\right)+\left[x(S)+\sum_{l=1}^{r} x\left(T^{j_{t_{l}}^{l}} \cup\left\{j_{t_{l}}^{l}\right\}\right)+\sum_{\substack{l=1 \\
l \notin 亠 I}}^{k-1} x\left(S_{l}\right)-x(N)\right] \\
& =v(N)-x(N)+x(S) \\
& =v\left(S_{t}\right)-x\left(N \backslash\left\{i_{k}\right\}\right)+x\left(S \backslash\left\{i_{k}\right\}\right)
\end{aligned}
$$

where the first inequality follows by reordering the terms in the inequality derived above; the first equality follows because $v\left(S_{l}\right)=x\left(S_{l}\right)$ for every $l \in\{1, \ldots, k-1\}$ by definition of $x_{i_{l}}$ (see equation (A.6)); the second inequality follows by equation(A.9) and $v(R) \leq x(R)$ for every $R \in\left(\cup_{l=0}^{k} S_{l}\right) \backslash\left\{i_{k}\right\}$ by induction and Case 1 ; the second equality follows by both the definition of mc-balanced vector for $\tilde{C}$ and the definition of $x_{j_{t_{l}}^{l}}$ (see equation (A.5)) and $T^{j_{t_{l}}^{l}}$ (see equation (A.8)); the third equality is a direct consequence of equations (3.1) and (3.2); the last equality readily follows since $i_{k} \in S \cap N$.

\section{Proof of Theorem 3.2.}

First we show the "if" part which is inspired in the proof of Theorem 5 in Sharkey (1982). Let $(N, v)$ be a game satisfying all minimal cover inequalities. By Theorem 3.1 it follows that $(N, v)$ has a non-empty core. We have to show that $\mathrm{L}(v)=\operatorname{Core}(v)$. We proceed by contradiction. Assume on the contrary that $\mathrm{L}(v) \neq \operatorname{Core}(v)$, then there exists $x \in \mathrm{L}(v) \backslash \operatorname{Core}(v)$. Note that $x(N)>v(N)$.

First we show that for every $i \in N$ there exist $S \in 2^{N} \backslash\{\emptyset\}$ with $i \in S$ and $x(S)=v(S)$ by contradiction. Assume on the contrary that the there is $i \in N$ such that $x(S)>v(S)$ for every $S \in 2^{\mathrm{N}} \backslash\{\emptyset\}$ with $i \in S$. Then we can take $\epsilon>0$ and $x^{\epsilon}$ defined by $x_{j}^{\epsilon}=x_{j}$ for every $j \in N \backslash\{i\}$ and $x_{i}^{\epsilon}=x_{i}-\epsilon$ such that 
$x^{\epsilon}(S) \geq v(S)$ for every $S \in 2^{\mathrm{N}} \backslash\{\emptyset\}$. Since $x^{\epsilon} \leq x$ and $x^{\epsilon} \neq x$, we may conclude that $x \notin \mathrm{L}(v)$, establishing a contradiction.

For each $i \in N$, let $S_{i} \in 2^{\mathrm{N}} \backslash\{\emptyset\}$ be a coalition such that $i \in S_{i}$ and $x\left(S_{i}\right)=v\left(S_{i}\right)$. Note that: (i) $S_{i} \neq N$ for every $i \in N$ since $x \in \mathrm{L}(v) \backslash \operatorname{Core}(v)$ and (ii) $\cup_{i \in N} S_{i}=N$. It is readily seen that there is a minimal cover $C \subset\left\{S_{i}\right\}_{i \in N}$. Let $\left\{i_{1}, \ldots, i_{k}\right\} \subset N$ such that $C=\left\{S_{i}\right\}_{i \in N \backslash\left\{i_{1}, \ldots, i_{k}\right\}}$. Then, it follows

$$
\begin{aligned}
x(N) & =\sum_{S \in C} x(S)-\sum_{i \in E(C)} \xi(C, i) x_{i} \\
& =\sum_{S \in C} v(S)-\sum_{i \in E(C)} \xi(C, i) x_{i} \\
& \leq \sum_{S \in C} v(S)-\min \left\{\sum_{i \in E(C)} \xi(C, i) \tilde{x}_{i} \mid \tilde{x}(S) \geq v(S) \text { for all } S \subset E(C)\right\} \\
& \leq v(N)
\end{aligned}
$$

where the second equality is a direct consequence of how $C$ has been constructed, the first inequality follows by Lemma A.1 (i), and the second inequality is a direct consequence of all minimal cover inequalities being satisfied. Hence, this establishes a contradiction and therefore $\mathrm{L}(v)=\operatorname{Core}(v)$.

The "only if" part follows the same lines and arguments as the proof of Theorem 3.3.1 in van Velzen (2005). We provide it here for the sake of completeness.

Let $(N, v)$ be a balanced game with a large core. We have to show that all minimal cover inequalities are satisfied. We proceed by contradiction. Assume that there is a minimal cover $C$ which inequality is violated, i.e.

$$
\sum_{S \in C} v(S)>v(N)+\max _{\mu \in M(C)}\left\{\sum_{S \in 2^{N} \backslash\{\emptyset\}} \mu_{S} v(S)\right\} .
$$

We construct $x \in \mathrm{U}(v) \backslash \operatorname{Core}(v)$ such that there is no $y \in \operatorname{Core}(v)$ with $y \leq x$, contradicting the fact that $(N, v)$ has a large core. Let $\hat{x} \in \mathbb{R}^{E(C)}$ be a solution to $\min \left\{\sum_{i \in E(C)} \xi_{i}(C) \tilde{x}_{i} \mid \tilde{x}(S) \geq v(S)\right.$ for all $\left.S \subset E(C)\right\}$ and define $x \in \mathbb{R}^{N}$ by

$$
x_{i}= \begin{cases}\hat{x}_{i} & \text { if } i \in E(C) \\ v(N) & \text { if } i \in N \backslash E(C) .\end{cases}
$$

It easily follows that $x \in \mathrm{U}(v)$ since for $S \in 2^{\mathrm{N}} \backslash\{\emptyset\}$, we have that $x(S)=\hat{x}(S) \geq v(S)$ if $S \subset E(C)$ and $x(S) \geq \hat{x}(S \cap E(C))+v(N) \geq v(S \cap E(C))+v(N) \geq v(S)$ if $S \not \subset E(C)$ where the first inequality follows by non-negativity of $(N, v)$, the second inequality follows by feasibility of $\hat{x}$, and the last inequality follows by non-negativity of $(N, v)$ and non-emptiness of the core of $(N, v)$.

Since $(N, v)$ has a large core, we can take $y \in \operatorname{Core}(v)$ such that $y \leq x$. Then,

$$
\sum_{S \in C} y(S)=y(N)+\sum_{i \in E(C)} \xi(C, i) y_{i}
$$




$$
\begin{aligned}
& =v(N)+\sum_{i \in E(C)} \xi(C, i) y_{i} \\
& \leq v(N)+\sum_{i \in E(C)} \xi(C, i) x_{i} \\
& =v(N)+\sum_{i \in E(C)} \xi(C, i) \hat{x}_{i} \\
& \left.=v(N)+\min _{i \in E(C)} \xi(C, i) \tilde{x}_{i} \mid \sum_{i \in S} \tilde{x}_{i} \geq v(S) \text { for all } S \in E(C)\right\} \\
& =v(N)+\max _{\mu \in M(C)}\left\{\sum_{S \in 2^{N} \backslash\{\emptyset\}} \mu_{S} v(S)\right\} \\
& <\sum_{S \in C} v(S)
\end{aligned}
$$

where the second equality follows because $y \in \operatorname{Core}(v)$, the third equality follows by definition of $x$, the fourth equality follows by definition of $\hat{x}$, the fifth equality follows by Lemma A.1 (i) and the last inequality follows by equation (A.10). Hence, it follows that $\sum_{S \in C} y(S)<\sum_{S \in C} v(S)$. We may conclude that there is $S \in C$ with $y(S)<v(S)$, which contradicts $y \in \operatorname{Core}(v)$.

\section{Largeness of the core and basic solutions}

In this part of the appendix, we show the two main results in Subsection 3.2. For this, we first provide two auxiliary lemmas.

The following result gives some relations between the weights that a base assigns to a coalition and the weights that the same base assigns to its members. It follows straightforward from the fact that $e^{R}=$ $\sum_{i \in R} e^{\{i\}}$ and therefore the proof is omitted.

Lemma A.2. Let $(N, v)$ be a game and let $B \in \mathbf{B}(N)$. Then,

(i) $\eta^{R}(S)=\sum_{i \in R} \eta^{\{i\}}(S)$ for every $R \in 2^{\mathrm{N}} \backslash\{\emptyset\}$ and every $S \in B$,

(ii) $x_{i}^{B}=\sum_{S \in B} \eta^{\{i\}}(S) v(S)$ for every $i \in N$,

(iii) $x^{B}(R)=\sum_{S \in B} \eta^{R}(S) v(S)$ for every $R \in 2^{\mathrm{N}} \backslash\{\emptyset\}$,

(iv) $x^{B, \alpha}(R)=\left\{\begin{array}{ll}x^{B}(R)+\alpha \eta^{R}(N) & \text { if } N \in B, \\ x^{B}(R) & \text { if } N \notin B,\end{array}\right.$ for every $R \in 2^{\mathrm{N}} \backslash\{\emptyset\}$.

The next result states that if $B \in \mathbf{B}(N)$ provides an element $x^{B, \alpha}(\alpha>0)$ of the upper core of a game $(N, v)$ such that $x^{B, \alpha} \geq x$ for some core element $x$, then $x$ is induced by $B$. 
Lemma A.3. Let $(N, v)$ be a balanced game and let $B \in \mathbf{B}(N)$ be such that $x^{B, \alpha} \in \mathrm{U}(v)$ for some $\alpha>0$. If there exist $x \in \operatorname{Core}(v)$ with $x \leq x^{B, \alpha}$, then $x=x^{B}$.

Proof: Let $x \in \operatorname{Core}(v)$ be such that $x \leq x^{B, \alpha}$. Then $x(N)=v(N)$ and for $S \in B \backslash\{N\}$ it follows

$$
v(S) \leq x(S) \leq x^{B, \alpha}(S)=v^{\alpha}(S)=v(S)
$$

and therefore $x(S)=v(S)$. Here, the first inequality is a direct consequence of $x \in \operatorname{Core}(v)$, the second inequality follows by assumption, the first equality is a direct consequence of the definition of $x^{B, \alpha}$, and the last inequality follows by definition of $v^{\alpha}$ together with $S \neq N$. Therefore, $x(S)=v(S)$ for every $S \in B$. Thus it follows that $x=x^{B}$ by uniqueness of solution to the system of linear equations $y(S)=v(S)$ for every $S \in B . \square$

Proof of Theorem 3.4. First we show the "only if" part. Let $(N, v)$ have a large core. We have to show that $\mathbf{B}_{+}(N, v) \supset \mathbf{B}\left(N, v^{\alpha}\right)$ for every $\alpha>0$. We proceed by contradiction. Suppose that there exists $\bar{\alpha}>0$ such that $\mathbf{B}\left(N, v^{\alpha}\right) \not \subset \mathbf{B}_{+}(N, v)$ and let $B \in \mathbf{B}\left(N, v^{\alpha}\right) \backslash \mathbf{B}_{+}(N, v)$.

If $B \in \mathbf{B}_{0}(N)$, then $x^{B, \bar{\alpha}}=x^{B}$ by Lemma A.2 (iv). By largeness of the core, there exists $y \in \operatorname{Core}(v)$ such that $y \leq x^{B, \bar{\alpha}}$. By Lemma A.3 we have that $y=x^{B} \in \operatorname{Core}(v)$. This establishes a contradiction since $x^{B, \bar{\alpha}}=x^{B}$ with $x^{B, \bar{\alpha}} \in \operatorname{Core}\left(v^{\bar{\alpha}}\right)$ and $\bar{\alpha}>0$.

If $B \in \mathbf{B}_{-}(N)$, then there exists $i \in N$ such that $\eta^{\{i\}}(N)<0$ and $x_{i}^{B, \bar{\alpha}}=x_{i}^{B}+\bar{\alpha} \eta^{\{i\}}(N)<x_{i}^{B}$ by Lemma A.2 (iv). By largeness of the core, there exists $y \in \operatorname{Core}(v)$ such that $y \leq x^{B, \bar{\alpha}}$. By Lemma A.3 we have that $y=x^{B} \in \operatorname{Core}(v)$. This establishes a contradiction since $x_{i}^{B, \bar{\alpha}}<x_{i}^{B}$.

If $B \in \mathbf{B}_{+}(N)$, then $x^{B} \notin \operatorname{Core}(v)$ since $B \notin \mathbf{B}_{+}(N, v)$. By largeness of the core, there exists $y \in \operatorname{Core}(v)$ such that $y \leq x^{B, \bar{\alpha}}$. By Lemma A.3 we have that $y=x^{B}$, establishing a contradiction.

Next we show the "if" part. Let $(N, v)$ be a game with $\mathbf{B}_{+}(N, v) \supset \mathbf{B}\left(N, v^{\alpha}\right)$ for every $\alpha>0$. We have to show that $(N, v)$ has a large core. For this it suffices to show that for every $\alpha>0$ and every $B \in \mathbf{B}\left(N, v^{\alpha}\right)$, there exists $y \in \operatorname{Core}(v)$ with $y \leq x^{B, \alpha}$. By Lemma A.3, this is equivalent to show that $x^{B} \in \operatorname{Core}(v)$ and $x^{B} \leq x^{B, \alpha}$. Since $\mathbf{B}\left(N, v^{\alpha}\right) \subset \mathbf{B}_{+}(N, v)$ for every $\alpha>0$, it readily follows $x^{B} \in \operatorname{Core}(v)$ and $x^{B} \leq x^{B, \alpha}$ for every $\alpha>0$ and every $B \in \mathbf{B}\left(N, v^{\alpha}\right)$ by Lemma A.2 (iv).

Proof of Theorem 3.5. First we show the "only if" part. Let $(N, v)$ be a game with a large core. By Theorem 3.4 it follows that $\mathbf{B}_{+}(N, v) \supset \mathbf{B}\left(N, v^{\alpha}\right)$ for every $\alpha>0$. We show that (i), (ii), (iii), (iv) and (v) are satisfied.

(i) Since $\mathbf{B}_{+}(N, v) \supset \mathbf{B}\left(N, v^{\alpha}\right)$ for every $\alpha>0$ and there exist $\bar{\alpha}>0$ with $\mathbf{B}\left(N, v^{\alpha}\right) \neq \emptyset$, it readily follows that $\mathbf{B}_{+}(N, v) \neq \emptyset$. 
(ii) We proceed by contradiction. Suppose that there exists $B \in \mathbf{B}_{+}(N) \backslash \mathbf{B}_{+}(N, v)$ such that $x^{B}(R) \geq v(R)$ for every $R \in 2^{\mathrm{N}} \backslash\{\emptyset\}$ with $\eta^{R}(N)=0$. Since $B \in \mathbf{B}_{+}(N) \backslash \mathbf{B}_{+}(N, v)$ and therefore $x^{B} \notin \operatorname{Core}(v)$, there must be $R \in 2^{\mathrm{N}} \backslash\{\emptyset\}$ such that $\eta^{R}(N)>0$ and $x^{B}(R)<v(R)$. By Lemma A.2 (iv), we have that $x^{B, \alpha}(R)=x^{B}(R)+\alpha \eta^{R}(N)$ for every $R \in 2^{\mathrm{N}} \backslash\{\emptyset\}$. By fixing $\bar{\alpha} \geq \max \left\{v(R)-x^{B}(R) \mid R \in\right.$ $2^{\mathrm{N}} \backslash\{\emptyset\}$ with $\left.x^{B}(R)<v(R)\right\}$ it readily follows that $x^{B, \bar{\alpha}} \in \operatorname{Core}\left(v^{\bar{\alpha}}\right)$. Hence $B \in \mathbf{B}\left(N, v^{\bar{\alpha}}\right) \not \subset$ $\mathbf{B}_{+}(N, v)$, establishing a contradiction.

(iii) Since $\mathbf{B}_{+}(N, v) \supset \mathbf{B}\left(N, v^{\alpha}\right)$ for every $\alpha>0$, it follows that $\mathbf{B}_{0}\left(N, v^{\alpha}\right)=\emptyset$. Let $B \in \mathbf{B}_{0}(N)$ with $x^{B}(N)=v(N)+\alpha$ for some $\alpha>0$. By Lemma A.2 (iv) we have that $x^{B}=x^{B, \alpha}$. Since $B \notin \mathbf{B}\left(N, v^{\alpha}\right)$, there exists $R \in 2^{N} \backslash\{\emptyset, N\}$ such that $v(R)>x^{B, \alpha}(R)=x^{B}(R)$.

(iv) We proceed by contradiction. Suppose that there exists $B \in \mathbf{B}_{-}(N) \backslash \mathbf{B}_{-}(N, v)$ such that (a), (b), and (c) are not satisfied. Then we have that

(a') $x^{B}(R) \geq v(R)$ for every $R \in 2^{\mathrm{N}} \backslash\{\emptyset\}$ with $\eta^{R}(N)=0$,

(b') $x^{B}(R)>v(R)$ for every $R \in 2^{\mathrm{N}} \backslash\{\emptyset\}$ with $\eta^{R}(N)<0$, and

(c') $\min _{\substack{R \in 2^{N} \backslash\{\emptyset\} \\ \eta^{R}(N)<0}}\left\{\max \left\{0, \frac{1}{\left|\eta^{R}(N)\right|}\left(x^{B}(R)-v(R)\right)\right\}\right\} \geq \max _{\substack{R \in 2^{N} \backslash\{\emptyset\} \\ \eta^{R}(N)>0}}\left\{\frac{1}{\eta^{R}(N)}\left(v(R)-x^{B}(R)\right)\right\}$.

Note that $\frac{1}{\left|\eta^{R}(N)\right|}\left(x^{B}(R)-v(R)\right)>0$ for every $R \in 2^{\mathrm{N}} \backslash\{\emptyset\}$ with $\eta^{R}(N)<0$ by (b'). Moreover, since $B \in \mathbf{B}_{-}(N) \backslash \mathbf{B}_{-}(N, v)$ we have that $x^{B} \notin \operatorname{Core}(v)$. By (a') and (b') it follows that there exists $R \in 2^{\mathrm{N}} \backslash\{\emptyset\}$ with $\eta^{R}(N)>0$ and $v(R)>x^{B}(R)$. Fix $\bar{\alpha}=\min _{\substack{R \in 2^{\mathrm{N}} \backslash\{\emptyset\} \\ \eta^{R}(N)<0}}\left\{\max \left\{0, \frac{1}{\left|\eta^{R}(N)\right|}\left(x^{B}(R)-\right.\right.\right.$ $v(R))\}\}$, next we show that $x^{B, \bar{\alpha}} \in \operatorname{Core}\left(v^{\bar{\alpha}}\right)$.

By Lemma A.2 (iv), we have that $x^{B, \bar{\alpha}}(R)=x^{B}(R)+\bar{\alpha} \eta^{R}(N)$ for every $R \in 2^{\mathrm{N}} \backslash\{\emptyset\}$. Then $x^{B, \bar{\alpha}}(N)=$ $x^{B}(N)+\bar{\alpha} \eta^{N}(N)=v(N)+\bar{\alpha}=v^{\bar{\alpha}}(N)$ and efficiency follows. Next, we show coalitional rationality. Let $R \in 2^{N} \backslash\{\emptyset, N\}$. If $\eta^{R}(N)=0$, then $x^{B, \bar{\alpha}}(R)=x^{B}(R) \geq v(R)$ by (a'). If $\eta^{R}(N)<0$, then

$$
\begin{aligned}
x^{B, \bar{\alpha}}(R) & =x^{B}(R)+\bar{\alpha} \eta^{R}(N) \\
& =x^{B}(R)+\min _{\substack{\tilde{R} \in 2^{\mathbb{N}} \backslash\{\emptyset\} \\
\eta^{\tilde{R}}(N)<0}}\left\{\max \left\{0, \frac{1}{\left|\eta^{\tilde{R}}(N)\right|}\left(x^{B}(\tilde{R})-v(\tilde{R})\right)\right\}\right\} \eta^{R}(N) \\
& =x^{B}(R)+\min _{\substack{\tilde{R} \in 2^{\mathrm{N}} \backslash\{\emptyset\} \\
\eta^{\tilde{R}}(N)<0}}\left\{\frac{1}{\left|\eta^{\tilde{R}}(N)\right|}\left(x^{B}(\tilde{R})-v(\tilde{R})\right)\right\} \eta^{R}(N) \\
& \geq x^{B}(R)+\frac{\eta^{R}(N)}{\left|\eta^{R}(N)\right|}\left(x^{B}(R)-v(R)\right) \\
& =x^{B}(R)-\left(x^{B}(R)-v(R)\right)=v(R)
\end{aligned}
$$


where the second equality follows by definition of $\bar{\alpha}$, the third one is a direct consequence of (b'), the inequality follows because $\eta^{R}(N)<0$, and the fourth equality is also a direct consequence of $\eta^{R}(N)<0$. If $\eta^{R}(N)>0$ and $x^{B}(R) \geq v(R)$, then $x^{B, \bar{\alpha}}(R)=x^{B}(R)+\bar{\alpha} \eta^{R}(N)>x^{B}(R) \geq v(R)$. If $\eta^{R}(N)>0$ and $x^{B}(R)<v(R)$, then

$$
\begin{aligned}
& x^{B, \bar{\alpha}}(R)=x^{B}(R)+\bar{\alpha} \eta^{R}(N) \\
&=x^{B}(R)+\min _{\substack{\tilde{R} \in 2^{N} \backslash\{\emptyset\} \\
\eta^{\tilde{R}}(N)<0}}\left\{\max \left\{0, \frac{1}{\left|\eta^{\tilde{R}}(N)\right|}\left(x^{B}(\tilde{R})-v(\tilde{R})\right)\right\}\right\} \eta^{R}(N) \\
& \geq x^{B}(R)+\max _{\substack{\tilde{R} \in 2^{N} \backslash\{\emptyset\} \\
\eta^{\tilde{R}}(N)>0}}\left\{\frac{1}{\eta^{\tilde{R}}(N)}\left(v(\tilde{R})-x^{B}(\tilde{R})\right)\right\} \eta^{R}(N) \\
& \geq x^{B}(R)+\frac{\eta^{R}(N)}{\eta^{R}(N)}\left(v(R)-x^{B}(R)\right) \\
&=x^{B}(R)+\left(v(R)-x^{B}(R)\right)=v(R)
\end{aligned}
$$

where the inequality follows by ( $\left.\mathrm{c}^{\prime}\right)$. Therefore, $x^{B, \bar{\alpha}} \in \operatorname{Core}\left(v^{\bar{\alpha}}\right)$ and $B \in \mathbf{B}_{-}\left(N, v^{\bar{\alpha}}\right)$, establishing a contradiction.

(v) We proceed by contradiction. Assume that there exists $B \in \mathbf{B}_{-}(N, v)$ satisfying $x^{B}(R)>v(R)$ for every $R \in 2^{\mathrm{N}} \backslash\{\emptyset\}$ with $\eta^{R}(N)<0$. Define $\bar{\alpha}=\min _{\substack{R \in 2^{N} \backslash\{\emptyset\} \\ \eta^{R}(N)<0}}\left\{\frac{1}{\left|\eta^{R}(N)\right|}\left(x^{B}(R)-v(R)\right)\right\}$. Note that $\bar{\alpha}>0$. Next we show that $x^{B, \bar{\alpha}} \in \operatorname{Core}\left(v^{\bar{\alpha}}\right)$.

By Lemma A.2 (iv), we have that $x^{B, \bar{\alpha}}(R)=x^{B}(R)+\bar{\alpha} \eta^{R}(N)$ for every $R \in 2^{\mathrm{N}} \backslash\{\emptyset\}$. Then $x^{B, \bar{\alpha}}(N)=$ $x^{B}(N)+\bar{\alpha} \eta^{N}(N)=v(N)+\bar{\alpha}=v^{\bar{\alpha}}(N)$ and efficiency follows. Next, we show coalitional rationality. Let $R \in 2^{N} \backslash\{\emptyset, N\}$. If $\eta^{R}(N) \geq 0$, then $x^{B, \bar{\alpha}}(R)=x^{B}(R)+\bar{\alpha} \eta^{R}(N) \geq x^{B}(R) \geq v(R)$ where the last inequality follows because $x^{B} \in \operatorname{Core}(v)$. If $\eta^{R}(N)<0$, then

$$
\begin{aligned}
x^{B, \bar{\alpha}}(R) & =x^{B}(R)+\bar{\alpha} \eta^{R}(N) \\
& =x^{B}(R)+\min _{\substack{\tilde{R} \in \in^{N} \backslash\{\emptyset\} \\
\eta^{\tilde{R}}(N)<0}}\left\{\frac{1}{\left|\eta^{\tilde{R}}(N)\right|}\left(x^{B}(\tilde{R})-v(\tilde{R})\right)\right\} \eta^{R}(N) \\
& \geq x^{B}(R)+\frac{\eta^{R}(N)}{\left|\eta^{R}(N)\right|}\left(x^{B}(R)-v(R)\right) \\
& =x^{B}(R)-\left(x^{B}(R)-v(R)\right)=v(R)
\end{aligned}
$$

where the inequality and the third equality follow because $\eta^{R}(N)<0$. Therefore, $x^{B, \bar{\alpha}} \in \operatorname{Core}\left(v^{\bar{\alpha}}\right)$ and $B \in \mathbf{B}_{-}\left(N, v^{\bar{\alpha}}\right)$, establishing a contradiction.

Next we show the "if" part. Let $(N, v)$ be a game satisfying (i), (ii), (iii), (iv) and (v). We have to show that 
$(N, v)$ has a large core. By Theorem 3.4 it suffices to show that $\mathbf{B}\left(N, v^{\alpha}\right) \subset \mathbf{B}_{+}(N, v)$ for every $\alpha>0$. Note that $\mathbf{B}_{+}(N, v) \neq \emptyset$ by $(\mathrm{i})$.

Next we show that $\mathbf{B}_{0}\left(N, v^{\alpha}\right)=\emptyset$ for every $\alpha>0$. By (iii), if $B \in \mathbf{B}_{0}(N)$ with $x^{B}(N)>v(N)$, then there exists $R \in 2^{\mathrm{N}} \backslash\{\emptyset\}$ such that $x^{B}(R)<v(R)$. By Lemma A.2 (iv) we have that $x^{B, \alpha}=x^{B}$ for every $\alpha>0$. Hence $B \notin \mathbf{B}_{0}\left(N, v^{\alpha}\right)$ for every $\alpha>0$ and it readily follows that $\mathbf{B}_{0}\left(N, v^{\alpha}\right)=\emptyset$ for every $\alpha>0$.

Next we show that $\mathbf{B}_{-}\left(N, v^{\alpha}\right)=\emptyset$ for every $\alpha>0$. If $B \in \mathbf{B}_{-}(N, v)$, then there exists $R \in 2^{\mathrm{N}} \backslash\{\emptyset\}$ such that $\eta^{R}(N)<0$ and $x^{B}(R)=v(R)$ by $(\mathrm{v})$. By Lemma A.2 (iv), we have that $x^{B, \alpha}(R)=x^{B}(R)+\alpha \eta^{R}(N)<$ $x^{B}(R)=v(R)$ for every $\alpha>0$. Hence $B \notin \mathbf{B}_{-}\left(N, v^{\alpha}\right)$ for every $\alpha>0$.

If $B \in \mathbf{B}_{-}(N) \backslash \mathbf{B}_{-}(N, v)$, then at least one of the conditions (a), (b), and (c) is satisfied by (iv). If (a) is satisfied, then there exists $R \in 2^{\mathrm{N}} \backslash\{\emptyset\}$ such that $\eta^{R}(N)=0$ and $x^{B}(R)<v(R)$. By Lemma A.2 (iv) we have that $x^{B, \alpha}(R)=x^{B}(R)+\alpha \eta^{R}(N)=x^{B}(R)<v(R)$ for every $\alpha>0$. Hence $B \notin \mathbf{B}_{-}\left(N, v^{\alpha}\right)$ for every $\alpha>0$. If (b) is satisfied, then there exists $R \in 2^{\mathrm{N}} \backslash\{\emptyset\}$ such that $\eta^{R}(N)<0$ and $x^{B}(R) \leq v(R)$. By Lemma A.2 (iv) we have that $x^{B, \alpha}(R)=x^{B}(R)+\alpha \eta^{R}(N)<x^{B}(R) \leq v(R)$ for every $\alpha>0$. Hence $B \notin \mathbf{B}_{-}\left(N, v^{\alpha}\right)$ for every $\alpha>0$. If (a) and (b) are not satisfied, then (c) is satisfied. Besides, there exists $R \in 2^{\mathrm{N}} \backslash\{\emptyset\}$ such that $\eta^{R}(N)>0$ and $x^{B}(R)<v(R)$ since $B \notin \mathbf{B}_{-}(N, v)$. Fix

$$
\bar{R} \in \arg \max _{\substack{R \in 2^{\mathrm{N}} \backslash\{\emptyset\} \\ \eta^{R}(N)>0}}\left\{\frac{1}{\eta^{R}(N)}\left(v(R)-x^{B}(R)\right)\right\} \text { and } \tilde{R} \in \arg \min _{\substack{R \in 2^{\mathrm{N}} \backslash\{\emptyset\} \\ \eta^{R}(N)<0}}\left\{\max \left\{0, \frac{1}{\left|\eta^{R}(N)\right|}\left(x^{B}(R)-v(R)\right)\right\}\right\} .
$$

Let $0<\alpha<\frac{1}{\eta^{\bar{R}}(N)}\left(v(\bar{R})-x^{B}(\bar{R})\right)$. By Lemma A.2 (iv) we have that $x^{B, \alpha}(\bar{R})=x^{B}(\bar{R})+\alpha \eta^{\bar{R}}(N)<$ $x^{B}(\bar{R})+\frac{\eta^{\bar{R}}(N)}{\eta^{\bar{R}}(N)}\left(v(\bar{R})-x^{B}(\bar{R})\right)=v(\bar{R})$ and therefore $B \notin \mathbf{B}_{-}\left(N, v^{\alpha}\right)$.

Let $\alpha \geq \frac{1}{\eta^{R}(N)}\left(v(\bar{R})-x^{B}(\bar{R})\right)$. By (c) we have that $\alpha>\frac{1}{\left|\eta^{\tilde{R}}(N)\right|}\left(x^{B}(\tilde{R})-v(\tilde{R})\right)$. By Lemma A.2 (iv) we have that $x^{B, \alpha}(\tilde{R})=x^{B}(\tilde{R})+\alpha \eta^{\tilde{R}}(N)<x^{B}(\tilde{R})+\frac{\eta^{\tilde{R}}(N)}{\left|\eta^{\tilde{R}}(N)\right|}\left(x^{B}(\tilde{R})-v(\tilde{R})\right)=x^{B}(\tilde{R})-\left(x^{B}(\tilde{R})-v(\tilde{R})\right)=v(\tilde{R})$ where the inequality follows because $\eta^{\tilde{R}}(N)<0$. Hence $B \notin \mathbf{B}_{-}\left(N, v^{\alpha}\right)$. Consequently, $\mathbf{B}_{-}\left(N, v^{\alpha}\right)=\emptyset$.

Therefore, $\mathbf{B}\left(N, v^{\alpha}\right)=\mathbf{B}_{+}\left(N, v^{\alpha}\right)$ for every $\alpha>0$. Finally, we show that $\mathbf{B}_{+}\left(N, v^{\alpha}\right) \subset \mathbf{B}_{+}(N, v)$ for every $\alpha>0$. By (ii), if $B \in \mathbf{B}_{+}(N) \backslash \mathbf{B}_{+}(N, v)$, then there exists $R \in 2^{\mathrm{N}} \backslash\{\emptyset\}$ such that $\eta^{R}(N)=0$ and $x^{B}(R)<v(R)$. By Lemma A.2 (iv) we have that $x^{B, \alpha}(R)=x^{B}(R)+\alpha \eta^{R}(N)=x^{B}(R)<v(R)$ for every $\alpha>0$. Hence $B \notin \mathbf{B}_{+}\left(N, v^{\alpha}\right)$ for every $\alpha>0$. Therefore $\mathbf{B}_{+}\left(N, v^{\alpha}\right) \subset \mathbf{B}_{+}(N, v)$ for every $\alpha>0$.

\section{Largeness of the core and stability}

In this part of the appendix, we show the main result in Section 4. Before starting the proof of Theorem 4.1, we pay attention to the structure of the minimal covers for games with at most 5 players. If $N=\{1,2\}$, then there is only one minimal cover $C=\{\{1\},\{2\}\}$, and $|E(C)|=0$. If $N=\{1,2,3\}$, then a minimal cover $C \in \mathcal{M C}(N)$ satisfies $|E(C)| \leq 1$; if $|E(C)|=1$ then $C$ is of the form: $\{\{i, j\},\{i, k\}\}$ with $\{i, j, k\}=N$. If 
$N=\{1,2,3,4\}$, then a minimal cover $C \in \mathcal{M C C}(N)$ satisfies $|E(C)| \leq 2$; if $|E(C)|=2$ then $C$ is of the form: $\{\{i, j, k\},\{i, j, l\}\}$, with $\{i, j, k, l\}=N$. If $N=\{1,2,3,4,5\}$, then a minimal cover $C \in \mathcal{M C C}(N)$ satisfies $|E(C)| \leq 3$; if $|E(C)|=3$ then $C$ is of the form: $\{\{i, j, k, l\},\{i, j, k, m\}\}$, with $\{i, j, k, l, m\}=N$.

Proof of Theorem 4.1. Let $(N, v)$ be a game with $|N| \leq 5$. It is well known that if $(N, v)$ has a large core, then the core is stable (see Theorem 3 in Sharkey (1982)). Therefore, we only have to show that if $(N, v)$ has a stable core, then the core is large. Note that non-emptiness of the core is equivalent to both largeness of the core and stability of the core for $|N|=2$. Therefore, we only have to show the result for $|N|>2$.

We proceed by contradiction. We suppose that $(N, v)$ has a stable core that is not large. Then we construct $x \in \mathrm{I}(v) \backslash \operatorname{Core}(v)$ in such a way that there is no $y \in \operatorname{Core}(v)$ and $S \in 2^{\mathrm{N}} \backslash\{\emptyset\}$ with $x(S)<v(S)$ such that $y(S)=v(S)$ and $x_{i}<y_{i}$ for every $i \in S$. This establishes a contradiction since $(N, v)$ has a stable core.

Fix $C \in \mathcal{M C}(N)$ such that

$$
\sum_{S \in C} v(S)>v(N)+\max _{\mu \in M(C)}\left\{\sum_{S \in 2^{N} \backslash\{\emptyset\}} \mu_{S} v(S)\right\} .
$$

It follows that $|E(C)|>0$ by non-emptiness of the core. We assume without loss of generality that $1 \in E(C)$.

Let $\tilde{z} \in \operatorname{Core}(v)$, then

$$
\begin{aligned}
\sum_{S \in C} v(S) & \leq \sum_{S \in C} \tilde{z}(S)=\tilde{z}(N)+\sum_{i \in E(C)} \xi(C, i) \tilde{z}_{i}=v(N)+\sum_{i \in E(C)} \xi(C, i) \tilde{z}_{i} \\
& <\sum_{S \in C} v(S)-\max _{\mu \in M(C)}\left\{\sum_{S \in 2^{N} \backslash\{\emptyset\}} \mu_{S} v(S)\right\}+\sum_{i \in E(C)} \xi(C, i) \tilde{z}_{i}
\end{aligned}
$$

and therefore

$$
\sum_{i \in E(C)} \xi(C, i) \tilde{z}_{i}>\max _{\mu \in M(C)}\left\{\sum_{S \in 2^{N} \backslash\{\emptyset\}} \mu_{S} v(S)\right\}
$$

for every $\tilde{z} \in \operatorname{Core}(v)$. As a result, for each $\tilde{z} \in \operatorname{Core}(v)$ there exists at least one $i \in E(C)$ such that $\tilde{z}_{i}>v(\{i\})$. Then, we fix $z \in \operatorname{Core}(v)$ satisfying

$$
z \in \arg \min _{\tilde{z} \in \operatorname{Core}(v)}\left\{\sum_{S \in 2^{\mathbb{N}} \backslash\{\emptyset\}} \xi(C, i) \tilde{z}_{i}\right\} .
$$

We distinguish between three cases: $|E(C)|=1,|E(C)|=2$, and $|E(C)|=3$.

Case 1: $|E(C)|=1$.

Recall that $1 \in E(C)$ and therefore $z_{1}>v(\{1\})$ by equation (A.11).

Fix $0<\varepsilon \leq \min \left\{z(R)-v(R) \mid R \in 2^{N}, 1 \in R, z(R)>v(R)\right\}$ and define $x \in \mathbb{R}^{N}$ as $x_{1}=z_{1}-\varepsilon, x_{2}=z_{2}+\varepsilon$, and $x_{j}=z_{j}$ for every $j \in N \backslash\{1,2\}$. It readily follows that $x \in \mathrm{I}(v) \backslash$ Core $(v)$ by selection of $\varepsilon, z_{1}>v(\{1\})$, 
and equation (A.12). Note that for $R \in 2^{\mathrm{N}} \backslash\{\emptyset\}$ with $x(R)<v(R)$ we have that $z(R)=v(R), 1 \in R$, and $2 \notin R$ by definition of $x$ and selection of $\varepsilon$.

By stability of the core, there exist $y \in \operatorname{Core}(v)$ and $S \in 2^{\mathrm{N}} \backslash\{\emptyset\}$ with $x(S)<v(S)$ such that $y(S)=v(S)$ and $y_{j}>x_{j}$ for every $j \in S$. Define $\beta \in \mathbb{R}^{N}$ as $\beta=y-x$. It readily follows that $\beta(N)=0, \beta(S)=\varepsilon$, and $\beta_{j}>0$ for every $j \in S$. Moreover, $\beta_{1} \geq \varepsilon$ since $y \in \operatorname{Core}(v)$ and therefore $y_{1} \geq z_{1}=x_{1}+\varepsilon$, where the inequality follows by equation (A.12) and the equality follows by definition of $x$. Note that $1 \in S$ and $S \neq\{1\}$, hence

$$
\varepsilon=\beta(S)=\beta_{1}+\beta(S \backslash\{1\})>\beta_{1} \geq \varepsilon
$$

establishing a contradiction.

As a result, if $(N, v)$ does not have a large core, then there must be a minimal cover $C$ with $|E(C)|>1$ that does not satisfy the minimal cover inequality. Consequently, we show that stability of the core implies largeness of the core for $|N|=3$.

Case 2: $|E(C)|=2$.

Assume without loss of generality that $E(C)=\{1,2\}$ and $\xi(C, 1) \geq \xi(C, 2)$. We distinguish between two subcases: $|N|=4$ and $|N|=5$.

Subcase 2.1: $|N|=4$.

Note that $C=\{\{1,2,3\},\{1,2,4\}\}$ and therefore $\xi(C, 1)=\xi(C, 2)$. By equation (A.11) there exists $i \in\{1,2\}$ such that $z_{i}>v(\{i\})$. Assume without loss of generality that $z_{1}>v(\{1\})$.

Fix $0<\varepsilon \leq \min \left\{z(R)-v(R) \mid R \in 2^{N}, 1 \in R, z(R)>v(R)\right\}$ and define, for $i \in N \backslash\{1,2\}, x^{i} \in \mathbb{R}^{N}$ as $x_{1}^{i}=z_{1}-\varepsilon, x_{i}^{i}=z_{i}+\varepsilon$, and $x_{j}^{i}=z_{j}$ for $j \in N \backslash\{1, i\}$. For $i \in N \backslash\{1,2\}$, it readily follows that $x^{i} \in \mathrm{I}(v) \backslash \operatorname{Core}(v)$ by selection of $\varepsilon, z_{1}>v(\{1\})$, and equation (A.12). Note that for $i \in N \backslash\{1,2\}$ and $R \in 2^{\mathrm{N}} \backslash\{\emptyset\}$ with $x^{i}(R)<v(R)$ we have that $z(R)=v(R), 1 \in R$, and $i \notin R$ by definition of $x^{i}$ and selection of $\varepsilon$.

By stability of the core, for $i \in N \backslash\{1,2\}$, there exist $y^{i} \in \operatorname{Core}(v)$ and $S^{i} \in 2^{\mathrm{N}} \backslash\{\emptyset\}$ with $x^{i}\left(S^{i}\right)<v\left(S^{i}\right)$ such that $y^{i}\left(S^{i}\right)=v\left(S^{i}\right)$ and $y_{j}^{i}>x_{j}^{i}$ for every $j \in S^{i}$. Define $\beta^{i} \in \mathbb{R}^{N}$ as $\beta^{i}=y^{i}-x^{i}$. It readily follows that $\beta^{i}(N)=0, \beta^{i}\left(S^{i}\right)=\varepsilon$, and $\beta_{j}^{i}>0$ for every $j \in S^{i}$. Moreover for $i \in N \backslash\{1,2\}$, it readily follows that $\beta^{i}(\{1,2\}) \geq \varepsilon$ since $y^{i} \in \operatorname{Core}(v)$ and therefore $y^{i}(\{1,2\}) \geq z(\{1,2\})=x^{i}(\{1,2\})+\varepsilon$, where the inequality follows by equation (A.12) and the equality follows by definition of $x^{i}$. Note that for $i \in N \backslash\{1,2\}, S^{i} \neq\{1,2\}$ by equation (A.11). Hence, if $2 \in S^{i}$, then $S^{i} \backslash\{1,2\} \neq \emptyset$ and

$$
\varepsilon=\beta^{i}\left(S^{i}\right)=\beta^{i}(\{1,2\})+\beta^{i}\left(S^{i} \backslash\{1,2\}\right)>\beta^{i}(\{1,2\}) \geq \varepsilon
$$

establishing a contradiction. Therefore $\{1,2\} \not \subset S^{i}$ for $i \in N \backslash\{1,2\}$. Hence, it readily follows that $S^{3}=\{1,4\}$, 
$S^{4}=\{1,3\}$ and then $z(\{1,3\})=v(\{1,3\})$ and $z(\{1,4\})=v(\{1,4\})$. Therefore, $\beta^{3}(\{1,4\})=\varepsilon, \beta^{3}(\{1,2\}) \geq \varepsilon$, $\beta^{3}(\{1,3\}) \geq 0$ and $\beta^{3}(N)=0$. Hence,

$$
2 \varepsilon \leq \beta^{3}(\{1,4\})+\beta^{3}(\{1,2\})+\beta^{3}(\{1,3\})=2 \beta_{1}^{3}<2 \beta^{3}(\{1,4\})=2 \varepsilon
$$

establishing a contradiction.

As a result, if $(N, v)$ does not have a large core, then there must be a minimal cover $C$ with $|E(C)|>2$ that does not satisfy the minimal cover inequality. However, $|N|=4$ and therefore $|E(C)| \leq 2$ for every $C \in \mathcal{M C}(N)$. Consequently, we show that stability of the core implies largeness of the core for $|N|=4$.

Subcase 2.2: $|N|=5$.

We consider two new subcases: $\xi(C, 1)=\xi(C, 2)$ and $\xi(C, 1)>\xi(C, 2)$.

Subcase 2.2.1: $\xi(C, 1)=\xi(C, 2)$.

By equation (A.11) there exists $i \in\{1,2\}$ such that $z_{i}>v(\{i\})$. Assume without loss of generality that $z_{1}>v(\{1\})$.

Fix $0<\varepsilon \leq \min \left\{z(R)-v(R) \mid R \in 2^{N}, 1 \in R, z(R)>v(R)\right\}$ and define, for $i \in N \backslash\{1,2\}, x^{i} \in \mathbb{R}^{N}$ as $x_{1}^{i}=z_{1}-\varepsilon, x_{i}^{i}=z_{i}+\varepsilon$, and $x_{j}^{i}=z_{j}$ for $j \in N \backslash\{1, i\}$. For $i \in N \backslash\{1,2\}$, it readily follows that $x^{i} \in \mathrm{I}(v) \backslash \operatorname{Core}(v)$ by selection of $\varepsilon, z_{1}>v(\{1\})$, and equation (A.12). Note that for $i \in N \backslash\{1,2\}$, if $R \in 2^{\mathrm{N}} \backslash\{\emptyset\}$ with $x^{i}(R)<v(R)$, we have that $z(R)=v(R), 1 \in R$, and $i \notin R$ by definition of $x^{i}$ and selection of $\varepsilon$.

By stability of the core, for $i \in N \backslash\{1,2\}$, there exist $y^{i} \in \operatorname{Core}(v)$ and $S^{i} \in 2^{\mathrm{N}} \backslash\{\emptyset\}$ with $x^{i}\left(S^{i}\right)<v\left(S^{i}\right)$ such that $y^{i}\left(S^{i}\right)=v\left(S^{i}\right)$ and $y_{j}^{i}>x_{j}^{i}$ for every $j \in S^{i}$. Define $\beta^{i} \in \mathbb{R}^{N}$ as $\beta^{i}=y^{i}-x^{i}$. It readily follows that $\beta^{i}(N)=0, \beta^{i}\left(S^{i}\right)=\varepsilon$, and $\beta_{j}^{i}>0$ for every $j \in S^{i}$. Moreover for $i \in N \backslash\{1,2\}, \beta^{i}(\{1,2\}) \geq \varepsilon$ since $y^{i} \in \operatorname{Core}(v)$ and therefore $y^{i}(\{1,2\}) \geq z(\{1,2\})=x^{i}(\{1,2\})+\varepsilon$, where the inequality follows by equation (A.12) and the equality follows by definition of $x^{i}$. Besides, it is easily seen that, for $i \in N \backslash\{1,2\}$ and $R \in 2^{\mathrm{N}} \backslash\{\emptyset\}, z(R)>v(R)$ in the following situations:

$$
\begin{array}{ll}
\bullet 1, i \in R \text { and } \beta^{i}(R)<0 ; & \bullet 1 \in R, i \notin R, \text { and } \beta^{i}(R)<\varepsilon ; \\
\text { - } i \in R, 1 \notin R \text {, and } \beta^{i}(R)<-\varepsilon ; & \bullet 1, i \notin R \text { and } \beta^{i}(R)<0 .
\end{array}
$$

Note that for $i \in N \backslash\{1,2\}, S^{i} \neq\{1,2\}$ by equation (A.11). Hence, if $2 \in S^{i}$, then $S^{i} \backslash\{1,2\} \neq \emptyset$ and

$$
\varepsilon=\beta^{i}\left(S^{i}\right)=\beta^{i}(\{1,2\})+\beta^{i}\left(S^{i} \backslash\{1,2\}\right)>\beta^{i}(\{1,2\}) \geq \varepsilon
$$

establishing a contradiction. Therefore $\{1,2\} \not \subset S^{i}$ for $i \in N \backslash\{1,2\}$. Hence, it readily follows that $S^{3} \in$ $\{\{1,4\},\{1,5\},\{1,4,5\}\}, S^{4} \in\{\{1,3\},\{1,5\},\{1,3,5\}\}$, and $S^{5} \in\{\{1,3\},\{1,4\},\{1,3,4\}\}$. 
- Let $S^{3}=\{1,4,5\}$, then $\beta^{3}(\{1,4,5\})=\varepsilon$ and $\beta_{j}^{3}>0$ for every $j \in\{1,4,5\}$. Moreover, $\beta_{2}^{3} \geq \beta^{3}(\{4,5\})>0$ since $\beta^{3}(\{1,2\}) \geq \varepsilon$. Note that

$$
\begin{aligned}
& z(R)>v(R) \text { for } R \in\{\{1,4\},\{1,5\}\} \text { since } \beta^{3}(R)<\beta^{3}(\{1,4,5\})=\varepsilon ; \\
& z(R)>v(R) \text { for } R \in\{\{1,3\},\{1,3,4\},\{1,3,5\}\} \text { since } \beta^{3}(R)=-\beta^{3}(N \backslash R)<0 .
\end{aligned}
$$

Equations (A.13) and (A.14) establish a contradiction with $S^{4} \in\{\{1,3\},\{1,5\},\{1,3,5\}\}$ and $S^{5} \in\{\{1,3\},\{1,4\}$, $\{1,3,4\}\}$. Hence, $S^{3} \neq\{1,4,5\}$ and analogously $S^{4} \neq\{1,3,5\}$ and $S^{5} \neq\{1,3,4\}$. Therefore, $S^{3} \in$ $\{\{1,4\},\{1,5\}\}, S^{4} \in\{\{1,3\},\{1,5\}\}$, and $S^{5} \in\{\{1,3\},\{1,4\}\}$.

- Let $S^{3}=\{1,4\}$. If $z(\{1,3\})=v(\{1,3\})$, and $z(\{1,5\})=v(\{1,5\})$, then $\beta^{3}(\{1,3\}) \geq 0, \beta^{3}(\{1,4\})=\varepsilon$, $\beta^{3}(\{1,5\}) \geq \varepsilon, \beta^{3}(\{1,2\}) \geq \varepsilon$ and $\beta^{3}(N)=0$. Hence,

$$
3 \varepsilon \leq \beta^{3}(\{1,3\})+\beta^{3}(\{1,4\})+\beta^{3}(\{1,5\})+\beta^{3}(\{1,2\})=3 \beta_{1}^{3}<3 \beta^{3}(\{1,4\})=3 \varepsilon
$$

establishing a contradiction. Therefore $z(R)>v(R)$ for exactly one coalition $R \in\{\{1,3\},\{1,4\},\{1,5\}\}$.

Assume without loss of generality that $z(\{1,5\})>v(\{1,5\})$ and therefore $z(\{1,3\})=v(\{1,3\})$ (if $z(\{1,3\})>v(\{1,3\})$ then $z(\{1,5\})=v(\{1,5\})$ and we consider $\left.S^{5}=\{1,4\}\right)$. In this case, $S^{3}=\{1,4\}$ and $\beta^{3}(\{1,4\})=\varepsilon, \beta^{3}(\{1,3\}) \geq 0, \beta^{3}(\{1,2\}) \geq \varepsilon$ and $\beta^{3}(N)=0$. Note that $\beta_{2}^{3} \geq \beta_{4}^{3}>0$. Therefore $z_{5}>v(\{5\})$ since $\beta_{5}^{3}=-\beta^{3}(\{1,2,3,4\}) \leq-\beta^{3}(\{2,4\})<0$.

Fix $0<\tilde{\varepsilon} \leq \min \left\{\frac{z(R)-v(R)}{R \cap\{1,5\}|-| R \cap\{3,4\} \mid}\left|R \in 2^{N},\right| R \cap\{1,5\}|>| R \cap\{3,4\} \mid, z(R)>v(R)\right\}$ and define $\tilde{x} \in \mathbb{R}^{N}$ as $\tilde{x}_{j}=z_{j}-\tilde{\varepsilon}$ for $j \in\{1,5\}, \tilde{x}_{2}=z_{2}$, and $\tilde{x}_{j}=z_{j}+\tilde{\varepsilon}$ for $j \in\{3,4\}$. By selection of $\tilde{\varepsilon}, z_{1}>v(\{1\}), z_{5}>v(\{5\})$ and equation (A.12), it readily follows that $\tilde{x} \in \mathrm{I}(v) \backslash \operatorname{Core}(v)$. It is easily seen that if $R \in 2^{\mathrm{N}} \backslash\{\emptyset\}$ with $\tilde{x}(R)<v(R)$, then $z(R)=v(R)$ and $|R \cap\{1,5\}|>|R \cap\{3,4\}|$ by definition of $\tilde{x}$ and selection of $\tilde{\varepsilon}$. Note that

$$
\begin{aligned}
& z(\{2,5\})>v(\{2,5\}) \text { since } \beta^{3}(\{2,5\})=-\beta^{3}(\{1,3,4\}) \leq-\beta_{4}^{3}<0 ; \\
& z(R)>v(R) \text { for } R \in\{\{1,2,5\},\{1,4,5\},\{1,2,4,5\}\} \text { since } \beta^{3}(R)=-\beta^{3}(N \backslash R) \leq-\beta_{3}^{3} \leq \beta_{1}^{3}<\varepsilon ; \\
& z(R)>v(R) \text { for } R \in\{\{1,3,5\},\{1,2,3,5\}\} \text { since } \beta^{3}(R)=-\beta^{3}(N \backslash R)<0 .
\end{aligned}
$$

Since $z(\{1,2\})>v(\{1,2\})$ by equation (A.11) and by equations (A.15) to (A.17), it is easily seen that $\tilde{x} \in \operatorname{Core}(v)$, establishing a contradiction. Therefore $S^{3} \neq\{1,4\}$, which is in contradiction with $(N, v)$ having a stable core.

Subcase 2.2.2: $\xi(C, 1)>\xi(C, 2)$.

Note that $C=\{\{1,2, i\},\{1,2, j\},\{1, k\}\}$ with $\{i, j, k\}=N \backslash\{1,2\}$ and therefore $\xi(C, 1)=2$ and $\xi(C, 2)=1$. By equation (A.11) there exists $i \in\{1,2\}$ such that $z_{i}>v(\{i\})$. We distinguish between two new subcases: 
$z_{1}>v(\{1\})$ and $z_{1}=v(\{1\})$.

\section{Subcase 2.2.2.1: $z_{1}>v(\{1\})$.}

Fix $0<\varepsilon \leq \min \left\{z(R)-v(R) \mid R \in 2^{N}, 1 \in R, z(R)>v(R)\right\}$ and define $x \in \mathbb{R}^{N}$ as $x_{1}=z_{1}-\varepsilon, x_{2}=z_{2}+\varepsilon$, and $x_{j}=z_{j}$ for $j \in N \backslash\{1,2\}$. It readily follows that $x \in \mathrm{I}(v) \backslash$ Core $(v)$ by selection of $\varepsilon, z_{1}>v(\{1\})$, and equation (A.12). Note that if $R \in 2^{\mathrm{N}} \backslash\{\emptyset\}$ with $x(R)<v(R)$, then $z(R)=v(R), 1 \in R$, and $2 \notin R$ by definition of $x$ and selection of $\varepsilon$.

By stability of the core, there exist $y \in \operatorname{Core}(v)$ and $S \in 2^{\mathrm{N}} \backslash\{\emptyset\}$ with $x(S)<v(S)$ such that $y(S)=v(S)$ and $y_{j}>x_{j}$ for every $j \in S$. Define $\beta \in \mathbb{R}^{N}$ as $\beta=y-x$. It readily follows that $\beta(N)=0, \beta(S)=\varepsilon$, and $\beta_{j}>0$ for every $j \in S$. Moreover $2 \beta_{1}+\beta_{2} \geq \varepsilon$ since $y \in \operatorname{Core}(v)$ and therefore $2 y_{1}+y_{2} \geq 2 z_{1}+z_{2}=2 x_{1}+x_{2}+\varepsilon$, where the inequality follows by equation (A.12) and the equality follows by definition of $x$. Besides it is easily seen that, for $R \in 2^{\mathrm{N}} \backslash\{\emptyset\}, z(R)>v(R)$ in the following situations:

$$
\begin{array}{ll}
\bullet 1,2 \in R \text { and } \beta(R)<0 ; & \bullet 1 \in R, 2 \notin R \text {, and } \beta(R)<\varepsilon ; \\
\bullet 2 \in R, 1 \notin R \text {, and } \beta(R)<-\varepsilon ; & \bullet 1,2 \notin R \text { and } \beta(R)<0 .
\end{array}
$$

It readily follows that $S \in\{\{1,3\},\{1,4\},\{1,5\},\{1,3,4\},\{1,3,5\},\{1,4,5\},\{1,3,4,5\}\}$.

- Let $S=\{1,3,4,5\}$, then $\beta(\{1,3,4,5\})=\varepsilon$ and $\beta_{j}>0$ for every $j \in\{1,3,4,5\}$. Since $\beta(N)=0$, it follows $\beta_{2}=-\varepsilon$ establishing a contradiction with $2 \beta_{1}+\beta_{2} \geq \varepsilon$. Therefore $S \neq\{1,3,4,5\}$ and $S \in$ $\{\{1,3\},\{1,4\},\{1,5\},\{1,3,4\},\{1,3,5\},\{1,4,5\}\}$.

- Let $S=\{1,3,4\}$, then $\beta(\{1,3,4\})=\varepsilon$ and $\beta_{j}>0$ for every $j \in\{1,3,4\}$. Moreover, $\beta(\{1,2\}) \geq \beta(\{3,4\})>$ 0 since $2 \beta_{1}+\beta_{2} \geq \varepsilon$. It follows $z_{5}>v(\{5\})$ since $\beta_{5}=-\beta(\{1,2,3,4\})<0$.

Fix $0<\tilde{\varepsilon} \leq \min \left\{\frac{z(R)-v(R)}{|R \cap\{1,5\}|-|R \cap\{2,3\}|}\left|R \in 2^{N},\right| R \cap\{1,5\}|>| R \cap\{2,3\} \mid, z(R)>v(R)\right\}$ and define $\tilde{x} \in \mathbb{R}^{N}$ as $\tilde{x}_{j}=z_{j}-\tilde{\varepsilon}$ for $j \in\{1,5\}, \tilde{x}_{j}=z_{j}+\tilde{\varepsilon}$ for $j \in\{2,3\}$, and $\tilde{x}_{4}=z_{4}$. By selection of $\tilde{\varepsilon}, z_{1}>v(\{1\}), z_{5}>v(\{5\})$ and equation (A.12), it readily follows that $\tilde{x} \in \mathrm{I}(v) \backslash \operatorname{Core}(v)$. It is easily seen that if $R \in 2^{\mathrm{N}} \backslash\{\emptyset\}$ with $\tilde{x}(R)<v(R)$, then $z(R)=v(R)$ and $|R \cap\{1,5\}|>|R \cap\{2,3\}|$ by definition of $\tilde{x}$ and selection of $\tilde{\varepsilon}$. Note that

$$
\begin{aligned}
& z(\{1,4\})>v(\{1,4\}) \text { since } \beta(\{1,4\})<\beta(\{1,3,4\})=\varepsilon ; \\
& z(\{4,5\})>v(\{4,5\}) \text { since } \beta(\{4,5\})=-\beta(\{1,2,3\})<-\beta_{3}<0 ; \\
& z(R)>v(R) \text { for } R \in\{\{1,2,5\},\{1,2,4,5\}\} \text { since } \beta(R)=-\beta(N \backslash R) \leq-\beta_{3}<0 ; \\
& z(R)>v(R) \text { for } R \in\{\{1,5\},\{1,3,5\},\{1,4,5\},\{1,3,4,5\}\} \text { since } \beta(R)=-\beta(N \backslash R) \leq-\beta_{2}<\beta_{1}<\varepsilon .
\end{aligned}
$$

By equations (A.18) to (A.21) it is easily seen that $\tilde{x} \in \operatorname{Core}(v)$, establishing a contradiction. Hence $S \neq$ $\{1,3,4\}$ and analogously $S \neq\{1,3,5\}$ and $S \neq\{1,4,5\}$. Therefore $S \in\{\{1,3\},\{1,4\},\{1,5\}\}$. 
- Let $S=\{1,3\}$, then $\beta(\{1,3\})=\varepsilon, \beta_{1}>0$, and $\beta_{3}>0$. Moreover, $\beta(\{1,2\}) \geq \beta_{3}>0$ since $2 \beta_{1}+\beta_{2} \geq \varepsilon$. If $z(\{1,4\})=v(\{1,4\})$ and $z(\{1,5\})=v(\{1,5\})$, then $\beta(\{1,3\})=\varepsilon, \beta(\{1,4\}) \geq \varepsilon, \beta(\{1,5\}) \geq \varepsilon$, $\beta(\{1,2\})>0$ and $\beta(N)=0$. Hence,

$$
3 \varepsilon<\beta(\{1,3\})+\beta(\{1,4\})+\beta(\{1,5\})+\beta(\{1,2\})=3 \beta_{1}<3 \beta(\{1,3\})=3 \varepsilon
$$

establishing a contradiction. Therefore $z(\{1,4\})>v(\{1,4\})$ and/or $z(\{1,5\})>v(\{1,5\})$. Assume without loss of generality that $z(\{1,5\})>v(\{1,5\})$.

If $z(\{1,4\})=v(\{1,4\})$, then $\beta(\{1,3\})=\varepsilon, \beta(\{1,4\}) \geq \varepsilon, 2 \beta_{1}+\beta_{2} \geq \varepsilon$ and $\beta(N)=0$. Hence, $\beta(\{1,2\}) \geq$ $\beta_{3}>0, \beta_{4} \geq \beta_{3}>0$ and $\beta_{5}=-\beta(\{1,2,3,4\})<0$. Therefore, $z_{5}>v(\{5\})$.

Fix $0<\tilde{\varepsilon} \leq \min \left\{\frac{z(R)-v(R)}{2|R \cap\{5\}|+|R \cap\{1\}|-|R \cap\{2,3,4\}|}\left|R \in 2^{N}, 2\right| R \cap\{5\}|+| R \cap\{1\}|>| R \cap\{2,3,4\} \mid, z(R)>v(R)\right\}$ and define $\tilde{x} \in \mathbb{R}^{N}$ as $\tilde{x}_{1}=z_{1}-\tilde{\varepsilon}, \tilde{x}_{j}=z_{j}+\tilde{\varepsilon}$ for $j \in\{2,3,4\}$, and $\tilde{x}_{5}=z_{5}-2 \tilde{\varepsilon}$. By selection of $\tilde{\varepsilon}, z_{1}>v(\{1\})$, $z_{5}>v(\{5\})$ and equation (A.12), it readily follows that $\tilde{x} \in \mathrm{I}(v) \backslash \operatorname{Core}(v)$. Note that if $R \in 2^{\mathrm{N}} \backslash\{\emptyset\}$ with $\tilde{x}(R)<v(R)$, then $z(R)=v(R)$ and $2|R \cap\{5\}|+|R \cap\{1\}|>|R \cap\{2,3,4\}|$ by definition of $\tilde{x}$ and selection of $\tilde{\varepsilon}$. Note that

$$
\begin{aligned}
& z(R)>v(R) \text { for } R \in\{\{3,5\},\{4,5\}\} \text { since } \beta(R)=-\beta(N \backslash R)<-\beta(\{1,2\})<0 ; \\
& z(\{2,5\})>v(\{2,5\}) \text { since } \beta(\{2,5\})=-\beta(\{1,3,4\})=-\varepsilon-\beta_{4}<-\varepsilon ; \\
& z(R)>v(R) \text { for } R \in\{\{1,3,5\},\{1,4,5\},\{1,3,4,5\}\} \text { since } \beta(R)=-\beta(N \backslash R) \leq-\beta_{2}<\beta_{1}<\varepsilon ; \\
& z(R)>v(R) \text { for } R \in\{\{1,2,5\},\{1,2,3,5\},\{1,2,4,5\}\} \text { since } \beta(R)=-\beta(N \backslash R)<0 .
\end{aligned}
$$

By equations (A.22) to (A.25), it is easily seen that $\tilde{x} \in \operatorname{Core}(v)$, establishing a contradiction. Therefore $z(\{1,4\})>v(\{1,4\})$.

Recall that $\beta(\{1,3\})=\varepsilon$ and $\beta(\{1,2\}) \geq \beta_{3}>0$ since $2 \beta_{1}+\beta_{2} \geq \varepsilon$. Taking into account that $\beta(N)=0$, we have that

$$
z(\{4,5\})>v(\{4,5\}) \text { since } \beta(\{4,5\})=-\beta(\{1,2,3\})<-\beta_{3}<0 .
$$

Therefore, $\beta_{4}<0$ and/or $\beta_{5}<0$. Assume without loss of generality that $\beta_{5}<0$, then $z_{5}>v(\{5\})$.

If $\beta_{4} \geq 0$, fix $0<\bar{\varepsilon} \leq \min \left\{\frac{z(R)-v(R)}{|R \cap\{1,5\}|-|R \cap\{2,3\}|}\left|R \in 2^{N},\right| R \cap\{1,5\}|>| R \cap\{2,3\} \mid, z(R)>v(R)\right\}$ and define $\bar{x} \in \mathbb{R}^{N}$ as $\bar{x}_{j}=z_{j}-\bar{\varepsilon}$ for $j \in\{1,5\}, \bar{x}_{j}=z_{j}+\bar{\varepsilon}$ for $j \in\{2,3\}$, and $\bar{x}_{4}=z_{4}$. By selection of $\bar{\varepsilon}, z_{1}>v(\{1\})$, $z_{5}>v(\{5\})$ and equation (A.12), it readily follows that $\bar{x} \in \mathrm{I}(v) \backslash \operatorname{Core}(v)$. Note that if $R \in 2^{\mathrm{N}} \backslash\{\emptyset\}$ with $\bar{x}(R)<v(R)$, then $z(R)=v(R)$ and $|R \cap\{1,5\}|>|R \cap\{2,3\}|$ by definition of $\bar{x}$ and selection of $\bar{\varepsilon}$. Note that

$$
\begin{aligned}
& z(R)>v(R) \text { for } R \in\{\{1,4\},\{1,5\}\} \text { by assumption; } \\
& z(R)>v(R) \text { for } R \in\{\{1,2,5\},\{1,2,4,5\}\} \text { since } \beta(R)=-\beta(N \backslash R) \leq-\beta_{3}<0 ;
\end{aligned}
$$




$$
z(R)>v(R) \text { for } R \in\{\{1,3,5\},\{1,4,5\},\{1,3,4,5\}\} \text { since } \beta(R)=-\beta(N \backslash R) \leq-\beta_{2}<\beta_{1}<\varepsilon .
$$

By equations (A.26) to (A.29), it is easily seen that $\bar{x} \in \operatorname{Core}(v)$, establishing a contradiction. Therefore $\beta_{4}<0$ and $z_{4}>v(\{4\})$. Note that

$$
\beta_{4}>-\beta(\{1,2,3\}) \text { and } \beta_{5}>-\beta(\{1,2,3\})
$$

since $\beta(\{4,5\})=-\beta(\{1,2,3\}), \beta_{4}<0$, and $\beta_{5}<0$.

Fix $0<\hat{\varepsilon} \leq \min \left\{\frac{z(R)-v(R)}{2|R \cap\{1\}|+|R \cap\{4,5\}|-2|R \cap\{2,3\}|}\left|R \in 2^{N}, 2\right| R \cap\{1\}|+| R \cap\{4,5\}|>2| R \cap\{2,3\} \mid, z(R)>\right.$ $v(R)\}$ and define $\hat{x} \in \mathbb{R}^{N}$ as $\hat{x}_{1}=z_{1}-2 \hat{\varepsilon}, \hat{x}_{j}=z_{j}+2 \hat{\varepsilon}$ for $j \in\{2,3\}$, and $\hat{x}_{j}=z_{j}-\hat{\varepsilon}$ for $j \in\{4,5\}$. By selection of $\hat{\varepsilon}, z_{1}>v(\{1\}), z_{4}>v(\{4\}), z_{5}>v(\{5\})$ and equation (A.12), it readily follows that $\hat{x} \in \mathrm{I}(v) \backslash \operatorname{Core}(v)$. Note that

$$
\begin{aligned}
& z(R)>v(R) \text { for } R \in\{\{1,3,4\},\{1,3,5\}\} \text { since } \beta(R)=-\beta(N \backslash R)<-\beta_{2}+\beta(\{1,2,3\})=\beta(\{1,3\})=\varepsilon ; \\
& z(R)>v(R) \text { for } R \in\{\{1,4,5\},\{1,3,4,5\}\} \text { since } \beta(R)=-\beta(N \backslash R) \leq-\beta_{2}<\beta_{1}<\varepsilon \\
& z(\{1,2,4,5\})>v(\{1,2,4,5\}) \text { since } \beta(\{1,2,4,5\})=-\beta_{3}<0
\end{aligned}
$$

By equations (A.26), (A.27), and (A.30) to (A.32), it is easily seen that $z(R)=v(R)$ for at least one coalition $R \in\{\{1,2,4\},\{1,2,5\}\}$ since $\hat{x} \in \mathrm{I}(v) \backslash \operatorname{Core}(v)$.

By stability of the core, there exist $\hat{y} \in \operatorname{Core}(v)$ and $\hat{S} \in 2^{\mathrm{N}} \backslash\{\emptyset\}$ with $\hat{x}(\hat{S})<v(\hat{S})$ such that $\hat{y}(\hat{S})=v(\hat{S})$ and $\hat{y}_{j}>\hat{x}_{j}$ for every $j \in \hat{S}$. Define $\hat{\beta} \in \mathbb{R}^{N}$ as $\hat{\beta}=\hat{y}-\hat{x}$. It readily follows that $\hat{\beta}(N)=0, \hat{\beta}(\hat{S})=\hat{\varepsilon}$, and $\hat{\beta}_{j}>0$ for every $j \in \hat{S}$.

Assume without loss of generality that $\hat{S}=\{1,2,4\}$, then $\hat{\beta}(\{1,2,4\})=\hat{\varepsilon}$ and $\hat{\beta}(\{1,3\}) \geq 0$. If $z(\{1,2,5\})=v(\{1,2,5\})$, then $\hat{\beta}(\{1,2,5\}) \geq \hat{\varepsilon}$ and

$$
2 \hat{\varepsilon} \leq \hat{\beta}(\{1,2,4\})+\hat{\beta}(\{1,2,5\})+\hat{\beta}(\{1,3\})=2 \hat{\beta}_{1}+\hat{\beta}_{2}<2 \hat{\beta}(\{1,2,4\})=2 \hat{\varepsilon}
$$

establishing a contradiction. Therefore $z(\{1,2,5\})>v(\{1,2,5\})$. Fix

$0<\varepsilon^{\prime} \leq \min \left\{\frac{z(R)-v(R)}{2|R \cap\{1,5\}|+|R \cap\{4\}|-3|R \cap\{2\}|-2|R \cap\{3\}|}\left|R \in 2^{N}, 2\right| R \cap\{1,5\}|+| R \cap\{4\}|>3| R \cap\{2\}|+2| R \cap\{3\} \mid\right.$, $z(R)>v(R)\}$ and define $x^{\prime} \in \mathbb{R}^{N}$ as $x_{j}^{\prime}=z_{j}-2 \varepsilon^{\prime}$ for $j \in\{1,5\}, x_{2}^{\prime}=z_{2}+3 \varepsilon^{\prime}, x_{3}^{\prime}=z_{3}+2 \varepsilon^{\prime}, x_{4}^{\prime}=z_{4}-\varepsilon^{\prime}$. By selection of $\varepsilon^{\prime}, z_{1}>v(\{1\}), z_{4}>v(\{4\}), z_{5}>v(\{5\})$ and equation (A.12), it readily follows that $x^{\prime} \in \mathrm{I}(v) \backslash \operatorname{Core}(v)$. Note that

$$
\begin{aligned}
& z(\{1,2,5\})>v(\{1,2,5\}) \text { by assumption; } \\
& z(\{3,4,5\})>v(\{3,4,5\}) \text { since } \beta(\{3,4,5\})=-\beta(\{1,2\})<0 .
\end{aligned}
$$

By equations (A.26), (A.27), and (A.30) to (A.34), it is easily seen that $x^{\prime} \in$ Core $(v)$ establishing a contradiction. 
Therefore, if $C \in \mathcal{M C C}(N)$ with $E(C)=\{i, j\}, i \neq j$, and $\xi(C, i)>\xi(C, j)$, then for every $z \in$ $\arg \min _{\tilde{z} \in \operatorname{Core}(v)}\left\{\sum_{S \in 2^{N} \backslash\{\emptyset\}} \xi(C, i) \tilde{z}_{i}\right\}$ it must be $z_{i}=v(\{i\})$.

Subcase 2.2.2.2: $z_{1}=v(\{1\})$.

By equation (A.11) we have that $z_{2}>v(\{2\})$.

Fix $0<\varepsilon \leq \min \left\{z(R)-v(R) \mid R \in 2^{N}, 2 \in R, z(R)>v(R)\right\}$ and define, for $i \in N \backslash\{1,2\}, x^{i} \in \mathbb{R}^{N}$ as $x_{2}^{i}=z_{2}-\varepsilon, x_{i}^{i}=z_{i}+\varepsilon$, and $x_{j}^{i}=z_{j}$ for $j \in N \backslash\{2, i\}$. For $i \in N \backslash\{1,2\}$, it readily follows that $x^{i} \in \mathrm{I}(v) \backslash \operatorname{Core}(v)$ by selection of $\varepsilon, z_{2}>v(\{2\})$, and equation (A.12). For $i \in N \backslash\{1,2\}$, note that if $R \in 2^{\mathrm{N}} \backslash\{\emptyset\}$ with $x^{i}(R)<v(R)$, then $z(R)=v(R), 2 \in R$, and $i \notin R$ by definition of $x^{i}$ and selection of $\varepsilon$.

By stability of the core, for $i \in N \backslash\{1,2\}$, there exist $y^{i} \in \operatorname{Core}(v)$ and $S^{i} \in 2^{\mathrm{N}} \backslash\{\emptyset\}$ with $x^{i}\left(S^{i}\right)<v\left(S^{i}\right)$ such that $y^{i}\left(S^{i}\right)=v\left(S^{i}\right)$ and $y_{j}^{i}>x_{j}^{i}$ for every $j \in S^{i}$. Define $\beta^{i} \in \mathbb{R}^{N}$ as $\beta^{i}=y^{i}-x^{i}$. It readily follows that $\beta^{i}(N)=0, \beta^{i}\left(S^{i}\right)=\varepsilon$, and $\beta_{j}^{i}>0$ for every $j \in S^{i}$. Moreover for $i \in N \backslash\{1,2\}, 2 \beta_{1}^{i}+\beta_{2}^{i} \geq \varepsilon$ since $y^{i} \in \operatorname{Core}(v)$ and therefore $2 y_{1}^{i}+y_{2}^{i} \geq 2 z_{1}+z_{2}=2 x_{1}^{i}+x_{2}^{i}+\varepsilon$, where the inequality follows by equation (A.12) and the equality follows by definition of $x^{i}$. Besides it is easily seen that for $i \in N \backslash\{1,2\}$ and $R \in 2^{\mathrm{N}} \backslash\{\emptyset\}$, $z(R)>v(R)$ in the following situations:

$$
\begin{array}{ll}
-2, i \in R \text { and } \beta^{i}(R)<0 ; & \bullet 2 \in R, i \notin R \text {, and } \beta^{i}(R)<\varepsilon ; \\
\text { - } i \in R, 2 \notin R \text {, and } \beta^{i}(R)<-\varepsilon ; & \bullet 2, i \notin R \text { and } \beta^{i}(R)<0 .
\end{array}
$$

Since $z(\{1,2\})>v(\{1,2\})$ by equation (A.11), it readily follows that $S^{3} \in\{\{2,4\},\{2,5\},\{1,2,4\},\{1,2,5\}$, $\{2,4,5\},\{1,2,4,5\}\}, S^{4} \in\{\{2,3\},\{2,5\},\{1,2,3\},\{1,2,5\},\{2,3,5\},\{1,2,3,5\}\}$, and $S^{5} \in\{\{2,3\},\{2,4\}$, $\{1,2,3\},\{1,2,4\},\{2,3,4\},\{1,2,3,4\}\}$.

- Let $S^{3}=\{1,2,4,5\}$, then $\beta^{3}(\{1,2,4,5\})=\varepsilon, \beta_{j}^{3}>0$ for every $j \in\{1,2,4,5\}$, and $\beta_{3}^{3}=-\varepsilon$. Note that

$$
\begin{aligned}
& z(R)>v(R) \text { for } R \in\{\{2,3\},\{1,2,3\},\{2,3,5\},\{1,2,3,5\}\} \text { since } \beta^{3}(R)=-\beta^{3}(N \backslash R) \leq-\beta_{4}^{3}<0 ; \\
& z(R)>v(R) \text { for } R \in\{\{2,3,4\},\{1,2,3,4\}\} \text { since } \beta^{3}(R)=-\beta^{3}(N \backslash R) \leq-\beta_{5}^{3}<0 ; \\
& z(R)>v(R) \text { for } R \in\{\{2,4\},\{2,5\},\{1,2,4\},\{1,2,5\}\} \text { since } \beta^{3}(R)=-\beta^{3}(N \backslash R)<-\beta_{3}^{3}=\varepsilon .
\end{aligned}
$$

Equations (A.35) to (A.37) establish a contradiction with $S^{4} \in\{\{2,3\},\{2,5\},\{1,2,3\},\{1,2,5\},\{2,3,5\}$, $\{1,2,3,5\}\}$ and $S^{5} \in\{\{2,3\},\{2,4\},\{1,2,3\},\{1,2,4\},\{2,3,4\},\{1,2,3,4\}\}$. Therefore $S^{3} \neq\{1,2,4,5\}$ and analogously $S^{4} \neq\{1,2,3,5\}$ and $S^{5} \neq\{1,2,3,4\}$. Then, $S^{3} \in\{\{2,4\},\{2,5\},\{1,2,4\},\{1,2,5\},\{2,4,5\}\}$, $S^{4} \in\{\{2,3\},\{2,5\},\{1,2,3\},\{1,2,5\},\{2,3,5\}\}$, and $S^{5} \in\{\{2,3\},\{2,4\},\{1,2,3\},\{1,2,4\},\{2,3,4\}\}$.

- Let $S^{3}=\{2,4,5\}$, then $\beta^{3}(\{2,4,5\})=\varepsilon$ and $\beta_{j}^{3}>0$ for every $j \in\{2,4,5\}$. Moreover, $\beta_{1}^{3} \geq \frac{1}{2} \beta^{3}(\{4,5\})>0$ since $2 \beta_{1}^{3}+\beta_{2}^{3} \geq \varepsilon$, and therefore $\beta_{3}^{3}=-\beta^{3}(\{1,2,4,5\})=-\varepsilon-\beta_{1}^{3}<-\varepsilon$. Note that

$$
z(R)>v(R) \text { for } R \in\{\{2,3\},\{1,2,3\},\{2,3,5\}\} \text { since } \beta^{3}(R)=-\beta^{3}(N \backslash R)<-\beta_{4}^{3}<0 ;
$$




$$
\begin{aligned}
& z(\{2,3,4\})>v(\{2,3,4\}) \text { since } \beta^{3}(\{2,3,4\})=-\beta^{3}(\{1,5\})<0 \\
& z(R)>v(R) \text { for } R \in\{\{2,4\},\{2,5\}\} \text { since } \beta^{3}(R)<\beta^{3}(\{2,4,5\})=\varepsilon
\end{aligned}
$$

By equations (A.38) to (A.40), it is easily seen that $S^{4}=\{1,2,5\}$ and $S^{5}=\{1,2,4\}$. Then, $z(\{1,2,4\})=$ $v(\{1,2,4\})$ and $z(\{1,2,5\})=v(\{1,2,5\})$.

Recall that $\beta_{3}^{3}=-\varepsilon-\beta_{1}^{3}<-\varepsilon$ and therefore $z_{3}>v(\{3\})$. Fix $0<\tilde{\varepsilon} \leq \min \left\{\frac{z(R)-v(R)}{2|R \cap\{2\}|+|R \cap\{3\}|-|R \cap\{1,4,5\}|}\left|R \in 2^{N}, 2\right| R \cap\{2\}|+| R \cap\{3\}|>| R \cap\{1,4,5\} \mid, z(R)>v(R)\right\}$ and define $\tilde{x} \in \mathbb{R}^{N}$ as $\tilde{x}_{j}=z_{j}+\tilde{\varepsilon}$ for $j \in\{1,4,5\}, \tilde{x}_{2}=z_{2}-2 \tilde{\varepsilon}$, and $\tilde{x}_{3}=z_{3}-\tilde{\varepsilon}$. By selection of $\tilde{\varepsilon}, z_{2}>v(\{2\})$, and $z_{3}>v(\{3\})$, it readily follows that $\tilde{x} \in \mathrm{I}(v)$. Note that

$$
\begin{aligned}
& z(\{1,2,3,4\})>v(\{1,2,3,4\}) \text { since } \beta^{3}(\{1,2,3,4\})=-\beta_{5}^{3}<0 \\
& z(\{1,2,3,5\})>v(\{1,2,3,5\}) \text { since } \beta^{3}(\{1,2,3,5\})=-\beta_{4}^{3}<0 \\
& z(\{2,3,4,5\})>v(\{2,3,4,5\}) \text { since } \beta^{3}(\{2,3,4,5\})=-\beta_{1}^{3}<0 .
\end{aligned}
$$

Since $z(\{1,2\})>v(\{1,2\})$ by equation (A.11) and by equations (A.38) to (A.43), it is easily seen that $\tilde{x} \in$ $\operatorname{Core}(v)$ and therefore we are in the conditions of Subcase 2.2.2.1, which establishes a contradiction. Hence, $S^{3} \neq\{2,4,5\}$ and analogously $S^{4} \neq\{2,3,5\}$ and $S^{5} \neq\{2,3,4\}$. Then, $S^{3} \in\{\{2,4\},\{2,5\},\{1,2,4\},\{1,2,5\}\}$, $S^{4} \in\{\{2,3\},\{2,5\},\{1,2,3\},\{1,2,5\}\}$, and $S^{5} \in\{\{2,3\},\{2,4\},\{1,2,3\},\{1,2,4\}\}$.

- Let $S^{3}=\{1,2,4\}$, then $\beta^{3}(\{1,2,4\})=\varepsilon$ and $\beta_{j}^{3}>0$ for every $j \in\{1,2,4\}$. Since $\beta^{3}(\{2,4\})<\varepsilon$, we have that

$$
z(\{2,4\})>v(\{2,4\})
$$

If $z(\{2,5\})=v(\{2,5\})$, then $\beta^{3}(\{1,2,4\})=\varepsilon, \beta^{3}(\{2,5\}) \geq \varepsilon, 2 \beta_{1}^{3}+\beta_{2}^{3} \geq \varepsilon$ and $\beta^{3}(N)=0$. Hence, $\beta_{5}^{3} \geq \beta^{3}(\{1,4\})>0$ and $\beta_{3}^{3}=-\beta^{3}(\{1,2,4,5\})<-\varepsilon$. Therefore $z_{3}>v(\{3\})$.

Fix $0<\tilde{\varepsilon} \leq \min \left\{\frac{z(R)-v(R)}{|R \cap\{2,3\}|-|R \cap\{4,5\}|}\left|R \in 2^{N},\right| R \cap\{2,3\}|>| R \cap\{4,5\} \mid, z(R)>v(R)\right\}$ and define $\tilde{x} \in \mathbb{R}^{N}$ as $\tilde{x}_{1}=z_{1}, \tilde{x}_{j}=z_{j}-\tilde{\varepsilon}$ for $j \in\{2,3\}, \tilde{x}_{j}=z_{j}+\tilde{\varepsilon}$ for $j \in\{4,5\}$. By selection of $\tilde{\varepsilon}, z_{2}>v(\{2\}), z_{3}>v(\{3\})$ and equation (A.12), it readily follows that $\tilde{x} \in \mathrm{I}(v) \backslash \operatorname{Core}(v)$. Note that,

$$
\begin{aligned}
& z(\{1,3\})>v(\{1,3\}) \text { since } \beta^{3}(\{1,3\})=-\beta^{3}(\{2,4,5\}) \leq-\varepsilon-\beta_{4}^{3}<-\varepsilon \\
& z(R)>v(R) \text { for } R \in\{\{2,3\},\{1,2,3\},\{2,3,4\},\{2,3,5\},\{1,2,3,4\},\{1,2,3,5\}\} \text { since } \\
& \beta^{3}(R)=-\beta^{3}(N \backslash R)<0 .
\end{aligned}
$$

Since $z(\{1,2\})>v(\{1,2\})$ by equation (A.11) and by equations (A.45) and (A.46), it is easily seen that $\tilde{x} \in \operatorname{Core}(v)$ which establishes a contradiction with $\tilde{x} \in \mathrm{I}(v) \backslash \operatorname{Core}(v)$. Therefore, $z(\{2,5\})>v(\{2,5\})$ and $S^{4} \in\{\{2,3\},\{1,2,5\},\{1,2,3\}\}$. 
If $z(\{1,2,5\})=v(\{1,2,5\})$, then $\beta^{3}(\{1,2,4\})=\varepsilon, \beta^{3}(\{1,2,5\}) \geq \varepsilon, 2 \beta_{1}^{3}+\beta_{2}^{3} \geq \varepsilon$ and $\beta^{3}(N)=0$. Hence, $\beta_{5}^{3} \geq \beta_{4}^{3}>0$ and $\beta_{3}^{3}=-\beta^{3}(\{1,2,4,5\})<-\varepsilon$. Therefore $z_{3}>v(\{3\})$.

Fix $0<\tilde{\varepsilon} \leq \min \left\{\frac{z(R)-v(R)}{2|R \cap\{2\}|+|R \cap\{3\}|-|R \cap\{1,4,5\}|}\left|R \in 2^{N}, 2\right| R \cap\{2\}|+| R \cap\{3\}|>| R \cap\{1,4,5\} \mid, z(R)>v(R)\right\}$ and define $\tilde{x} \in \mathbb{R}^{N}$ as $\tilde{x}_{j}=z_{j}+\tilde{\varepsilon}$ for $j \in\{1,4,5\}, \tilde{x}_{2}=z_{2}-2 \tilde{\varepsilon}$, and $\tilde{x}_{3}=z_{3}-\tilde{\varepsilon}$. By selection of $\tilde{\varepsilon}, z_{2}>v(\{2\})$, and $z_{3}>v(\{3\})$, it readily follows that $\tilde{x} \in \mathrm{I}(v)$. If $\tilde{x} \in \operatorname{Core}(v)$, then we are in the conditions of Subcase 2.2.2.1, which establishes a contradiction. Therefore $\tilde{x} \in \mathrm{I}(v) \backslash$ Core $(v)$. Note that if $R \in 2^{\mathrm{N}} \backslash\{\emptyset\}$ with $\tilde{x}(R)<v(R)$, then $z(R)=v(R)$ and $2|R \cap\{2\}|+|R \cap\{3\}|>|R \cap\{1,4,5\}|$ by definition of $\tilde{x}$ and selection of $\tilde{\varepsilon}$. Note that

$z(R)>v(R)$ for $R \in\{\{2,3\},\{1,2,3\},\{2,3,4\},\{2,3,5\},\{1,2,3,4\},\{1,2,3,5\},\{2,3,4,5\}\}$ since

$\beta^{3}(R)=-\beta^{3}(N \backslash R)<0$.

Since $\tilde{x} \in \mathrm{I}(v) \backslash \operatorname{Core}(v), z(\{1,2\})>v(\{1,2\})$ by equation (A.11), and by equations (A.44) and (A.47), it is easily seen that $z(\{2,5\})=v(\{2,5\})$. However, we have already seen that $z(\{2,5\})>v(\{2,5\})$ establishing a contradiction with $\tilde{x} \in \mathrm{I}(v) \backslash \operatorname{Core}(v)$. Therefore, $z(\{1,2,5\})>v(\{1,2,5\})$ and $S^{4} \in\{\{2,3\},\{1,2,3\}\}$.

If $z(\{2,3\})=v(\{2,3\})$, then $\beta^{3}(\{1,2,4\})=\varepsilon, \beta^{3}(\{2,3\}) \geq 0,2 \beta_{1}^{3}+\beta_{2}^{3} \geq \varepsilon$ and $\beta^{3}(N)=0$. Hence $z_{5}>v(\{5\})$ since $\beta_{5}^{3}=-\beta^{3}(\{1,2,3,4\}) \leq-\beta^{3}(\{1,4\})<0$. Fix

$0<\tilde{\varepsilon} \leq \min \left\{\frac{z(R)-v(R)}{|R \cap\{2,5\}|-|R \cap\{3,4\}|}\left|R \in 2^{N},\right| R \cap\{2,5\}|>| R \cap\{3,4\} \mid, z(R)>v(R)\right\}$ and define $\tilde{x} \in \mathbb{R}^{N}$ as $\tilde{x}_{1}=z_{1}, \tilde{x}_{j}=z_{j}-\tilde{\varepsilon}$ for $j \in\{2,5\}$, and $\tilde{x}_{j}=z_{j}+\tilde{\varepsilon}$ for $j \in\{3,4\}$. By selection of $\tilde{\varepsilon}, z_{2}>v(\{2\}), z_{5}>v(\{5\})$ and equation (A.12), it readily follows that $\tilde{x} \in \mathrm{I}(v) \backslash \operatorname{Core}(v)$. Note that if $R \in 2^{\mathrm{N}} \backslash\{\emptyset\}$ with $\tilde{x}(R)<v(R)$, then $z(R)=v(R)$ and $|R \cap\{2,5\}|>|R \cap\{3,4\}|$ by definition of $\tilde{x}$ and selection of $\tilde{\varepsilon}$. Moreover,

$$
\begin{aligned}
& z(\{1,5\})>v(\{1,5\}) \text { since } \beta^{3}(\{1,5\})=-\beta^{3}\left(\{2,3,4\} \leq-\beta_{4}^{3}<0\right. \\
& z(R)>v(R) \text { for } R \in\{\{2,3,5\},\{1,2,3,5\},\} \text { since } \beta^{3}(R)=-\beta^{3}(N \backslash R) \leq-\beta_{4}^{3}<0 \\
& z(R)>v(R) \text { for } R \in\{\{2,5\},\{1,2,5\},\{2,4,5\},\{1,2,4,5\}\} \text { since } \beta^{3}(R)=-\beta^{3}(N \backslash R) \leq-\beta_{3}^{3} \leq \beta_{2}^{3}<\varepsilon
\end{aligned}
$$

Since $z(\{1,2\})>v(\{1,2\})$ by equation (A.11) and by equations (A.48) to (A.50), it is easily seen that $\tilde{x} \in \operatorname{Core}(v)$, which establishes a contradiction with $\tilde{x} \in \mathrm{I}(v) \backslash \operatorname{Core}(v)$. Therefore $z(\{2,3\})>v(\{2,3\})$ and $S^{4}=\{1,2,3\}$. Then, $z(\{1,2,3\})=v(\{1,2,3\})$ and $\beta^{3}(\{1,2,4\})=\varepsilon, \beta^{3}(\{1,2,3\}) \geq 0,2 \beta_{1}^{3}+\beta_{2}^{3} \geq \varepsilon$ and $\beta^{3}(N)=0$. Hence $z_{5}>v(\{5\})$ since $\beta_{5}^{3}=-\beta^{3}(\{1,2,3,4\}) \leq-\beta_{4}^{3}<0$. Fix

$0<\tilde{\varepsilon} \leq \min \left\{\frac{z(R)-v(R)}{|R \cap\{2,5\}|-|R \cap\{3,4\}|}\left|R \in 2^{N},\right| R \cap\{2,5\}|>| R \cap\{3,4\} \mid, z(R)>v(R)\right\}$ and define $\tilde{x} \in \mathbb{R}^{N}$ as $\tilde{x}_{1}=z_{1}, \tilde{x}_{j}=z_{j}-\tilde{\varepsilon}$ for $j \in\{2,5\}$, and $\tilde{x}_{j}=z_{j}+\tilde{\varepsilon}$ for $j \in\{3,4\}$. By selection of $\tilde{\varepsilon}, z_{2}>v(\{2\}), z_{5}>v(\{5\})$ and equation (A.12), it readily follows that $\tilde{x} \in \mathrm{I}(v) \backslash \operatorname{Core}(v)$. Note that if $R \in 2^{\mathrm{N}} \backslash\{\emptyset\}$ with $\tilde{x}(R)<v(R)$, then $z(R)=v(R)$ and $|R \cap\{2,5\}|>|R \cap\{3,4\}|$ by definition of $\tilde{x}$ and selection of $\tilde{\varepsilon}$. Moreover,

$$
z(R)>v(R) \text { for } R \in\{\{2,3,5\},\{1,2,3,5\},\} \text { since } \beta^{3}(R)=-\beta^{3}(N \backslash R) \leq-\beta_{4}^{3}<0 ;
$$




$$
\begin{aligned}
& z(R)>v(R) \text { for } R \in\{\{2,5\},\{1,2,5\},\{2,4,5\},\{1,2,4,5\}\} \text { since } \\
& \beta^{3}(R)=-\beta^{3}(N \backslash R) \leq-\beta_{3}^{3} \leq \beta^{3}(\{1,2\})<\varepsilon .
\end{aligned}
$$

Since $z(\{1,2\})>v(\{1,2\})$ by equation (A.11) and by equations (A.51) and (A.52), together with $\tilde{x} \in$ $\mathrm{I}(v) \backslash \operatorname{Core}(v)$, it readily follows that $z(\{1,5\})=v(\{1,5\})$. Fix

$0<\bar{\varepsilon} \leq \min \left\{\frac{z(R)-v(R)}{2|R \cap\{2\}|+|R \cap\{5\}|-|R \cap\{1,3,4\}|}\left|R \in 2^{N}, 2\right| R \cap\{2\}|+| R \cap\{5\}|>| R \cap\{1,3,4\} \mid, z(R)>v(R)\right\}$ and define $\bar{x} \in \mathbb{R}^{N}$ as $\bar{x}_{j}=z_{j}+\bar{\varepsilon}$ for $j \in\{1,3,4\}, \bar{x}_{2}=z_{2}-2 \bar{\varepsilon}$, and $\bar{x}_{5}=z_{5}-\bar{\varepsilon}$. By selection of $\bar{\varepsilon}$, $z_{2}>v(\{2\})$, and $z_{5}>v(\{5\})$, it readily follows that $\bar{x} \in \mathrm{I}(v)$. If $\bar{x} \in \operatorname{Core}(v)$, we are in the conditions of Subcase 2.2.2.1, establishing a contradiction. Therefore, $\bar{x} \in \mathrm{I}(v) \backslash$ Core $(v)$. Since $z(\{1,5\})=v(\{1,5\})$ we have that $\beta^{3}(\{1,5\}) \geq 0$. Recall that $\beta^{3}(\{1,2,4\})=\varepsilon$ and $\beta^{3}(\{1,2,3\}) \geq 0$. Then,

$$
\begin{aligned}
& z(\{2,3\})>v(\{2,3\}) \text { since } \beta^{3}(\{2,3\})=-\beta^{3}(\{1,4,5\}) \leq-\beta_{4}^{3}<0 \\
& z(\{2,3,4,5\})>v(\{2,3,4,5\}) \text { since } \beta^{3}(\{2,3,4,5\})=-\beta_{1}^{3}<0 .
\end{aligned}
$$

Since $z(\{1,2\})>v(\{1,2\})$ by equation (A.11) and by equations (A.44) and (A.51) to (A.54), it is easily seen that $\bar{x} \in \operatorname{Core}(v)$ which establishes a contradiction with $\bar{x} \in \mathrm{I}(v) \backslash \operatorname{Core}(v)$. Hence, $z(\{1,2,3\})>v(\{1,2,3\})$ establishing a contradiction with $S^{4}=\{1,2,3\}$. Therefore, $S^{3} \neq\{1,2,4\}$ and analogously $S^{3} \neq\{1,2,5\}$, $S^{4} \notin\{\{1,2,3\},\{1,2,4\}\}$, and $S^{5} \notin\{\{1,2,3\},\{1,2,4\}\}$. Then, $S^{3} \in\{\{2,4\},\{2,5\}\}, S^{4} \in\{\{2,3\},\{2,5\}\}$, and $S^{5} \in\{\{2,3\},\{2,4\}\}$.

- Let $S^{3}=\{2,4\}$, then $\beta^{3}(\{2,4\})=\varepsilon$ and $\beta_{j}^{3}>0$ for every $j \in\{2,4\}$. Moreover, $2 \beta_{1}^{3}+\beta_{2}^{3} \geq \varepsilon$. Hence, $\beta_{1}^{3} \geq \frac{1}{2} \beta_{4}^{3}>0$.

If $z(\{2,3\})=v(\{2,3\})$ and $z(\{2,5\})=v(\{2,5\})$, then $\beta^{3}(\{2,3\}) \geq 0$ and $\beta^{3}(\{2,5\}) \geq \varepsilon$. Since $\beta_{1}^{3}>0$, $\beta^{3}(\{2,4\})=\varepsilon$, and $\beta^{3}(N)=0$, we have that

$$
2 \varepsilon<\beta_{1}^{3}+\beta^{3}(\{2,3\})+\beta^{3}(\{2,4\})+\beta^{3}(\{2,5\})=2 \beta_{2}^{3}<2 \beta^{3}(\{2,4\})=2 \varepsilon
$$

establishing a contradiction. Therefore $z(R)>v(R)$ for exactly one coalition $R \in\{\{2,3\},\{2,5\}\}$.

Assume without loss of generality that $z(\{2,5\})>v(\{2,5\})$, then $z(\{2,3\})=v(\{2,3\})$ and $\beta^{3}(\{2,3\}) \geq 0$. Since $\beta_{1}^{3}>0$ and $\beta^{3}(N)=0$ we have that $\beta_{5}^{3}=-\beta^{3}(\{1,2,3,4\}) \leq-\beta^{3}(\{1,4\})<0$ and therefore $z_{5}>v(\{5\})$.

Fix $0<\tilde{\varepsilon} \leq \min \left\{\frac{z(R)-v(R)}{|R \cap\{2,5\}|-|R \cap\{3,4\}|}\left|R \in 2^{N},\right| R \cap\{2,5\}|>| R \cap\{3,4\} \mid, z(R)>v(R)\right\}$ and define $\tilde{x} \in \mathbb{R}^{N}$ as $\tilde{x}_{1}=z_{1}, \tilde{x}_{j}=z_{j}-\tilde{\varepsilon}$ for $j \in\{2,5\}$, and $\tilde{x}_{j}=z_{j}+\tilde{\varepsilon}$ for $j \in\{3,4\}$. By selection of $\tilde{\varepsilon}, z_{2}>v(\{2\})$, $z_{5}>v(\{5\})$ and equation (A.12), it readily follows that $\tilde{x} \in \mathrm{I}(v) \backslash \operatorname{Core}(v)$. Note that if $R \in 2^{\mathrm{N}} \backslash\{\emptyset\}$ with $\tilde{x}(R)<v(R)$, then $z(R)=v(R)$ and $|R \cap\{2,5\}|>|R \cap\{3,4\}|$ by definition of $\tilde{x}$ and selection of $\tilde{\varepsilon}$. Recall that $\beta^{3}(\{2,4\})=\varepsilon, \beta^{3}(\{2,3\}) \geq 0$ and $\beta_{1}^{3}>0$. Then,

$$
z(\{1,5\})>v(\{1,5\}) \text { since } \beta^{3}(\{1,5\})=-\beta^{3}(\{2,3,4\}) \leq-\beta_{4}^{3}<0 ;
$$




$$
\begin{aligned}
& z(R)>v(R) \text { for } R \in\{\{2,3,5\},\{1,2,3,5\}\} \text { since } \beta^{3}(R)=-\beta^{3}(N \backslash R) \leq-\beta_{4}^{3}<0 ; \\
& z(R)>v(R) \text { for } R \in\{\{1,2,5\},\{2,4,5\},\{1,2,4,5\}\} \text { since } \beta^{3}(R)=-\beta^{3}(N \backslash R) \leq-\beta_{3}^{3} \leq \beta_{2}^{3}<\varepsilon .
\end{aligned}
$$

Since $z(\{1,2\})>v(\{1,2\})$ by equation (A.11) and by equations (A.55) to (A.57), it is easily seen that $\tilde{x} \in \operatorname{Core}(v)$ which establishes a contradiction with $\tilde{x} \in \mathrm{I}(v) \backslash \operatorname{Core}(v)$. Therefore $S^{3} \neq\{2,4\}$, which is in contradiction with $(N, v)$ having a stable core.

As a result, if $(N, v)$ does not have a large core, then there must be a minimal cover $C$ with $|E(C)|>2$ that does not satisfy the minimal cover inequality.

Case 3: $|E(C)|=3$.

Assume without loss of generality that $E(C)=\{1,2,3\}$. Note that $C=\{\{1,2,3,4\},\{1,2,3,5\}\}$ and therefore $\xi(C, 1)=\xi(C, 2)=\xi(C, 3)=1$. By equation (A.11) we have that

$$
\begin{aligned}
z(\{1,2,3\})>\max \{v(\{1\})+v(\{2\})+v(\{3\}), v(\{1\})+v(\{2,3\}), v(\{2\})+v(\{1,3\}), \\
\left.v(\{3\})+v(\{1,2\}), \frac{1}{2}(v(\{1,2\})+v(\{1,3\})+v(\{2,3\})), v(\{1,2,3\})\right\} .
\end{aligned}
$$

Therefore, there exists at least one player $i \in\{1,2,3\}$ with $z_{i}>v(\{i\})$ and players $j, k \in\{1,2,3\}$ with $j \neq k$ such that $z(\{j, k\})>v(\{j, k\})$. Moreover,

$$
z(\{1,2,3\})>v(\{1,2,3\})
$$

Let $\{1,2,3\}=\{i, j, k\}$, we distinguish between two subcases: $z(\{i, j\})=v(\{i, j\}), z(\{i, k\})=v(\{i, k\})$, and $z(\{j, k\})>v(\{j, k\}) ; z(\{i, j\}) \geq v(\{i, j\}), z(\{i, k\})>v(\{i, k\})$, and $z(\{j, k\})>v(\{j, k\})$.

Subcase 3.1: $z(\{i, j\})=v(\{i, j\}), z(\{i, k\})=v(\{i, k\})$, and $z(\{j, k\})>v(\{j, k\})$.

Assume without loss of generality that $z(\{1,2\})>v(\{1,2\})$ and therefore $z(\{1,3\})=v(\{1,3\})$ and $z(\{2,3\})=$ $v(\{2,3\})$. By equation (A.58) we have that $z_{1}>v(\{1\})$ and $z_{2}>v(\{2\})$. Fix

$0<\varepsilon \leq \min _{i \in\{4,5\}}\left\{\min \left\{\frac{z(R)-v(R)}{R \cap\{1,2\}|-| R \cap\{3, i\} \mid}\left|R \in 2^{N},\right| R \cap\{1,2\}|>| R \cap\{3, i\} \mid, z(R)>v(R)\right\}\right\}$ and define, for $i \in\{4,5\}, x^{i} \in \mathbb{R}^{N}$ as $x_{j}^{i}=z_{j}-\varepsilon$ for $j \in\{1,2\}, x_{j}^{i}=z_{j}+\varepsilon$ for $j \in\{3, i\}$, and $x_{j}^{i}=z_{j}$ for $j \in\{4,5\} \backslash\{i\}$. For $i \in\{4,5\}$, it readily follows that $x^{i} \in \mathrm{I}(v) \backslash \operatorname{Core}(v)$ by selection of $\varepsilon, z_{1}>v(\{1\}), z_{2}>v(\{2\})$ and equation (A.12). For $i \in\{4,5\}$, note that if $R \in 2^{\mathrm{N}} \backslash\{\emptyset\}$ with $x^{i}(R)<v(R)$, then $z(R)=v(R)$ and $|R \cap\{1,2\}|>|R \cap\{3, i\}|$ by definition of $x^{i}$ and selection of $\varepsilon$.

By stability of the core, there exist $y^{i} \in \operatorname{Core}(v)$ and $S^{i} \in 2^{\mathrm{N}} \backslash\{\emptyset\}$ with $x^{i}\left(S^{i}\right)<v\left(S^{i}\right)$ such that $y^{i}\left(S^{i}\right)=v\left(S^{i}\right)$ and $y_{j}^{i}>x_{j}^{i}$ for every $j \in S^{i}$. Define $\beta^{i} \in \mathbb{R}^{N}$ as $\beta^{i}=y^{i}-x^{i}$. It readily follows that $\beta^{i}(N)=0$, $\beta^{i}\left(S^{i}\right)=\varepsilon$, and $\beta_{j}^{i}>0$ for every $j \in S^{i}$. Moreover for $i \in N \backslash\{1,2\}, \beta^{i}(\{1,2,3\}) \geq \varepsilon$ since $y^{i} \in \operatorname{Core}(v)$ and therefore $y^{i}(\{1,2,3\}) \geq z(\{1,2,3\})=x^{i}(\{1,2,3\})+\varepsilon$, where the inequality follows by equation (A.12) 
and the equality follows by definition of $x^{i}$. Since $z(\{1,3\})=v(\{1,3\})$ and $z(\{2,3\})=v(\{2,3\})$, we also have that $\beta^{i}(\{1,3\}) \geq 0$ and $\beta^{i}(\{2,3\}) \geq 0$ for $i \in\{4,5\}$. Besides it is easily seen that for $i \in\{4,5\}$ and $R \in 2^{\mathrm{N}} \backslash\{\emptyset\}, z(R)>v(R)$ in the following situations:

- $|R \cap\{1,2\}|=|R \cap\{3, i\}|$ and $\beta^{i}(R)<0$;

- $|R \cap\{1,2\}|=|R \cap\{3, i\}|+1$ and $\beta^{i}(R)<\varepsilon ;$

- $|R \cap\{1,2\}|=|R \cap\{3, i\}|-2$ and $\beta^{i}(R)<-2 \varepsilon$;

- $|R \cap\{1,2\}|=|R \cap\{3, i\}|+2$ and $\beta^{i}(R)<2 \varepsilon$.

- $|R \cap\{1,2\}|=|R \cap\{3, i\}|-1$ and $\beta^{i}(R)<-\varepsilon$;

It readily follows that $S^{4} \in\{\{1,5\},\{2,5\},\{1,2,4\},\{1,2,5\},\{1,2,3,5\},\{1,2,4,5\}\}$ and $S^{5} \in\{\{1,4\},\{2,4\}$, $\{1,2,4\},\{1,2,5\},\{1,2,3,4\},\{1,2,4,5\}\}$.

- Let $S^{4}=\{1,2,4,5\}$, then $\beta^{4}(\{1,2,4,5\})=\varepsilon$ and $\beta_{j}^{4}>0$ for every $j \in\{1,2,4,5\}$. Recall that $\beta^{4}(\{1,3\}) \geq 0$, and $\beta^{4}(N)=0$. Hence,

$$
\varepsilon \leq \beta^{4}(\{1,2,4,5\})+\beta^{4}(\{1,3\})=\beta_{1}^{4}<\beta^{4}(\{1,2,4,5\})=\varepsilon
$$

establishing a contradiction. Therefore $S^{4} \neq\{1,2,4,5\}$ and analogously $S^{5} \neq\{1,2,4,5\}$. Then, $S^{4} \in$ $\{\{1,5\},\{2,5\},\{1,2,4\},\{1,2,5\},\{1,2,3,5\}\}$ and $S^{5} \in\{\{1,4\},\{2,4\},\{1,2,4\},\{1,2,5\},\{1,2,3,4\}\}$.

- Let $S^{4}=\{1,2,3,5\}$, then $\beta^{4}(\{1,2,3,5\})=\varepsilon$ and $\beta_{j}^{4}>0$ for every $j \in\{1,2,3,5\}$. Recall that $\beta^{4}(\{1,2,3\}) \geq$ $\varepsilon$. Hence

$$
\varepsilon \leq \beta^{4}(\{1,2,3\})<\beta^{4}(\{1,2,3,5\})=\varepsilon
$$

establishing a contradiction. Therefore $S^{4} \neq\{1,2,3,5\}$ and analogously $S^{5} \neq\{1,2,3,4\}$. Then, $S^{4} \in$ $\{\{1,5\},\{2,5\},\{1,2,4\},\{1,2,5\}\}$, and $S^{5} \in\{\{1,4\},\{2,4\},\{1,2,4\},\{1,2,5\}\}$.

- Let $S^{4}=\{1,2,4\}$, then $\beta^{4}(\{1,2,4\})=\varepsilon$ and $\beta_{j}^{4}>0$ for every $j \in\{1,2,4\}$. Recall that $\beta^{4}(\{1,3\}) \geq 0$, $\beta^{4}(\{2,3\}) \geq 0, \beta^{4}(\{1,2,3\}) \geq \varepsilon$ and $\beta^{4}(N)=0$. Hence, it is easily seen that $\beta_{3}^{4} \geq \beta_{4}^{4}>0$ and $\beta_{5}^{4}=$ $-\beta^{4}(\{1,2,3,4\})<0$. Therefore $z_{5}>v(\{5\})$.

Fix $0<\tilde{\varepsilon} \leq \min \left\{\frac{z(R)-v(R)}{|R \cap\{1,2,5\}|-|R \cap\{3\}|-2|R \cap\{4\}|}\left|R \in 2^{N},\right| R \cap\{1,2,5\}|>| R \cap\{3\}|+2| R \cap\{4\} \mid, z(R)>v(R)\right\}$ and define $\tilde{x} \in \mathbb{R}^{N}$ as $\tilde{x}_{j}=z_{j}-\tilde{\varepsilon}$ for $j \in\{1,2,5\}, \tilde{x}_{3}=z_{3}+\tilde{\varepsilon}$, and $\tilde{x}_{4}=z_{4}+2 \tilde{\varepsilon}$. By selection of $\tilde{\varepsilon}$, $z_{1}>v(\{1\}), z_{2}>v(\{2\}), z_{5}>v(\{5\})$ and equation (A.12), it readily follows that $\tilde{x} \in \mathrm{I}(v) \backslash \operatorname{Core}(v)$. Note that if $R \in 2^{\mathrm{N}} \backslash\{\emptyset\}$ with $\tilde{x}(R)<v(R)$, then $z(R)=v(R)$ and $|R \cap\{1,2,5\}|>|R \cap\{3\}|+2|R \cap\{4\}|$ by definition of $\tilde{x}$ and selection of $\tilde{\varepsilon}$. Note that,

$$
\begin{aligned}
& z(R)>v(R) \text { for } R \in\{\{1,5\},\{2,5\},\{1,2,3,5\}\} \text { since } \beta^{4}(R)=-\beta^{4}(N \backslash R) \leq-\beta_{4}^{4}<0<\varepsilon ; \\
& z(\{1,2,5\})>v(\{1,2,5\}) \text { since } \beta^{4}(\{1,2,5\})=-\beta^{4}(\{3,4\})<0<2 \varepsilon ; \\
& z(R)>v(R) \text { for } R \in\{\{1,3,5\},\{2,3,5\}\} \text { since } \beta^{4}(R)=-\beta^{4}(N \backslash R)<-\beta_{4}^{4}<0 ; \\
& z(\{1,2,4,5\})>v(\{1,2,4,5\}) \text { since } \beta^{4}(\{1,2,4,5\})=-\beta_{3}^{4}<0<\varepsilon .
\end{aligned}
$$


By equations (A.59) to (A.63), it is easily seen that $\tilde{x} \in \operatorname{Core}(v)$ which establishes a contradiction with $\tilde{x} \in$ $\mathrm{I}(v) \backslash \operatorname{Core}(v)$. Therefore $S^{4} \neq\{1,2,4\}$ and analogously $S^{5} \neq\{1,2,5\}$. Then, $S^{4} \in\{\{1,5\},\{2,5\},\{1,2,5\}\}$ and $S^{5} \in\{\{1,4\},\{2,4\},\{1,2,4\}\}$.

- Let $S^{4}=\{1,2,5\}$, then $\beta^{4}(\{1,2,5\})=2 \varepsilon$ and $\beta_{j}^{4}>0$ for every $j \in\{1,2,5\}$. Recall that $\beta^{4}(\{1,3\}) \geq 0$, $\beta^{4}(\{2,3\}) \geq 0, \beta^{4}(\{1,2,3\}) \geq \varepsilon$ and $\beta^{4}(N)=0$. Note that,

$$
\begin{aligned}
& z(R)>v(R) \text { for } R \in\{\{1,4\},\{2,4\}\} \text { since } \beta^{4}(R)=-\beta^{4}(N \backslash R) \leq-\beta_{5}^{4}<0<\varepsilon ; \\
& z(\{1,2,4\})>v(\{1,2,4\}) \text { since } \beta^{4}(\{1,2,4\})=-\beta^{4}(\{3,5\})<-\beta_{3}^{4} \leq \beta^{4}(\{1,2\})-\varepsilon<2 \varepsilon-\varepsilon=\varepsilon .
\end{aligned}
$$

Equations (A.64) and (A.65) establish a contradiction with $S^{5} \in\{\{1,4\},\{2,4\},\{1,2,4\}\}$. Therefore $S^{4} \neq$ $\{1,2,5\}$ and analogously $S^{5} \neq\{1,2,4\}$. Then, $S^{4} \in\{\{1,5\},\{2,5\}\}$, and $S^{5} \in\{\{1,4\},\{2,4\}\}$.

- Let $S^{4}=\{1,5\}$, then $\beta^{4}(\{1,5\})=\varepsilon$ and $\beta_{j}^{4}>0$ for every $j \in\{1,5\}$. Recall that $\beta^{4}(\{1,3\}) \geq 0$, $\beta^{4}(\{2,3\}) \geq 0, \beta^{4}(\{1,2,3\}) \geq \varepsilon$ and $\beta^{4}(N)=0$. Note that

$$
z(R)>v(R) \text { for } R \in\{\{1,4\},\{2,4\}\} \text { since } \beta^{3}(R)=-\beta^{3}(N \backslash R) \leq-\beta_{5}^{4}<0<\varepsilon .
$$

Equation (A.66) establishes a contradiction with $S^{5} \in\{\{1,4\},\{2,4\}\}$. Therefore $S^{4} \neq\{1,5\}$ and analogously $S^{4} \neq\{2,5\}$. This establishes a contradiction with $(N, v)$ being stable.

Subcase 3.2: $z(\{i, j\}) \geq v(\{i, j\}), z(\{i, k\})>v(\{i, k\})$, and $z(\{j, k\})>v(\{j, k\})$.

Assume without loss of generality that $z(\{1,2\})>v(\{1,2\}), z(\{1,3\})>v(\{1,3\})$, and $z(\{2,3\}) \geq v(\{2,3\})$. If $z(\{2,3\})=v(\{2,3\})$, then we have that $z_{1}>v(\{1\})$ by equation (A.58). If $z(\{2,3\})>v(\{2,3\})$, then we can assume without loss of generality that $z_{1}>v(\{1\})$ by equation (A.58). Fix

$0<\varepsilon \leq \min \left\{z(R)-v(R) \mid R \in 2^{N}, 1 \in R, z(R)>v(R)\right\}$ and define, for $i \in\{4,5\}, x^{i} \in \mathbb{R}^{N}$ as $x_{1}^{i}=z_{1}-\varepsilon$, $x_{i}^{i}=z_{i}+\varepsilon$, and $x_{j}^{i}=z_{j}$ for $j \in N \backslash\{1, i\}$. For $i \in\{4,5\}$, it readily follows that $x^{i} \in \mathrm{I}(v) \backslash \operatorname{Core}(v)$ by selection of $\varepsilon, z_{1}>v(\{1\})$, and equation (A.12). For $i \in\{4,5\}$, note that if $R \in 2^{\mathrm{N}} \backslash\{\emptyset\}$ with $x^{i}(R)<v(R)$, then $z(R)=v(R), 1 \in R$ and $i \notin R$ by definition of $x^{i}$ and selection of $\varepsilon$.

By stability of the core, there exist $y^{i} \in \operatorname{Core}(v)$ and $S^{i} \in 2^{\mathrm{N}} \backslash\{\emptyset\}$ with $x^{i}\left(S^{i}\right)<v\left(S^{i}\right)$ such that $y^{i}\left(S^{i}\right)=v\left(S^{i}\right)$ and $y_{j}^{i}>x_{j}^{i}$ for every $j \in S^{i}$. Define $\beta^{i} \in \mathbb{R}^{N}$ as $\beta^{i}=y^{i}-x^{i}$. It readily follows that $\beta^{i}(N)=0, \beta^{i}\left(S^{i}\right)=\varepsilon$, and $\beta_{j}^{i}>0$ for every $j \in S^{i}$. Moreover for $i \in\{4,5\}, \beta^{i}(\{1,2,3\}) \geq \varepsilon$ since $y^{i} \in \operatorname{Core}(v)$ and therefore $y^{i}(\{1,2,3\}) \geq z(\{1,2,3\})=x^{i}(\{1,2,3\})+\varepsilon$, where the inequality follows by equation (A.12) and the equality follows by definition of $x^{i}$. Besides it is easily seen that for $i \in\{4,5\}$ and $R \in 2^{\mathrm{N}} \backslash\{\emptyset\}, z(R)>v(R)$ in the following situations:

- $1, i \in R$ and $\beta^{i}(R)<0$;

- $1 \in R, i \notin R$, and $\beta^{i}(R)<\varepsilon$;

- $i \in R, 1 \notin R$, and $\beta^{i}(R)<-\varepsilon ; \quad \bullet 1, i \notin R$ and $\beta^{i}(R)<0$. 
It readily follows by equation (A.59) and the assumption $z(\{1,2\})>v(\{1,2\})$ and $z(\{1,3\})>v(\{1,3\})$ that $S^{4} \in\{\{1,5\},\{1,2,5\},\{1,3,5\},\{1,2,3,5\}\}$ and $S^{5} \in\{\{1,4\},\{1,2,4\},\{1,3,4\},\{1,2,3,4\}\}$.

- Let $S^{4}=\{1,2,3,5\}$, then $\beta^{4}(\{1,2,3,5\})=\varepsilon$ and $\beta_{j}^{4}>0$ for every $j \in\{1,2,3,5\}$. Recall that $\beta^{4}(\{1,2,3\}) \geq$ $\varepsilon$. Hence,

$$
\varepsilon \leq \beta^{4}(\{1,2,3\})<\beta^{4}(\{1,2,3,5\})=\varepsilon
$$

establishing a contradiction. Therefore $S^{4} \neq\{1,2,3,5\}$ and analogously $S^{5} \neq\{1,2,3,4\}$. Then, $S^{4} \in$ $\{\{1,5\},\{1,2,5\},\{1,3,5\}\}$, and $S^{5} \in\{\{1,4\},\{1,2,4\},\{1,3,4\}\}$.

- Let $S^{4}=\{1,3,5\}$, then $\beta^{4}(\{1,3,5\})=\varepsilon$ and $\beta_{j}^{4}>0$ for every $j \in\{1,3,5\}$. Recall that $\beta^{4}(\{1,2,3\}) \geq \varepsilon$ and therefore $\beta_{2}^{4} \geq \beta_{5}^{4}>0$. Note that,

$$
z(R)>v(R) \text { for } R \in\{\{1,4\},\{1,2,4\},\{1,3,4\}\} \text { since } \beta^{3}(R)=-\beta^{3}(N \backslash R)<0<\varepsilon .
$$

Equation (A.67) establishes a contradiction with $S^{5} \in\{\{1,4\},\{1,2,4\},\{1,3,4\}\}$. Therefore $S^{4} \neq\{1,3,5\}$ and analogously $S^{4} \neq\{1,2,5\}$ and $S^{5} \notin\{\{1,2,4\},\{1,3,4\}\}$. Therefore $S^{4}=\{1,5\}$ and $S^{5}=\{1,4\}$.

- Hence, $S^{4}=\{1,5\}$ and $\beta^{4}(\{1,5\})=\varepsilon$ with $\beta_{j}^{4}>0$ for every $j \in\{1,5\}$. Moreover, $z(\{1,4\})=v(\{1,4\})$ since $S^{5}=\{1,4\}$ and then $\beta^{4}(\{1,4\}) \geq 0$. Recall that $\beta^{4}(\{1,2,3\}) \geq \varepsilon$ and $\beta^{4}(N)=0$. Hence,

$$
2 \varepsilon \leq \beta^{4}(\{1,5\})+\beta^{4}(\{1,4\})+\beta^{4}(\{1,2,3\})=2 \beta_{1}^{4}<2 \beta^{4}(\{1,5\})=2 \varepsilon
$$

establishing a contradiction. This establishes a contradiction with $(N, v)$ being stable.

As a result, if $(N, v)$ does not have a large core, then there must be a minimal cover $C$ with $|E(C)|>3$ that does not satisfy the minimal cover inequality. However, $|E(C)| \leq 3$ for every minimal cover $C$ since $|N|=5$. Consequently, we show that stability of the core implies largeness of the core for $|N|=5$.

\section{References}

Azrieli, Y. and Lehrer, E. (2007), 'Extendable cooperative games', Journal of Public Economic Theory 9, 1069-1078.

Biswas, A. K., Parthasarathy, T., Potters, J. A. M. and Voorneveld, M. (1999), 'Large cores and exactness', Games and Economic Behavior 28, 1-12.

Biswas, A. K., Parthasarathy, T. and Ravindran, G. (2001), 'Stability and largeness of the core', Games and Economic Behavior 34, 227-237. 
Bondareva, O. N. (1963), 'Some applications of linear programming methods to the theory of cooperative games', Problemy Kibernitiki 10, 119-139 (in Russian).

Chvàtal, V. (1983), Linear Programming, W.H.Freeman, New York.

Csóka, P., Herings, P. and Kóczy, L. (2007), 'Balancedness conditions for exact games', METEOR Reserach Memorandum 07-40. Maastricht University, Maastricht, The Netherlands.

Dantzig, G. B. and Thapa, M. N. (2003), Linear Programming 2: Theory and Extensions, Series in Operations Research and Financial Engineering, Springer, New York.

Driessen, T. (1988), Cooperative Games, Solutions and Applications, Kluwer Academic Publishers.

Gillies, D. (1953), Some theorems on n-person games, PhD thesis, Princeton, University Press Princeton, New Jersey.

LeBreton, M., Owen, G. and Weber, S. (1992), 'Strongly balanced cooperative games', International Journal of Game Theory 20, 419-427.

Schmeidler, D. (1972), 'Cores of exact games', Journal of Mathematical Analysis and Applications 40, 214225.

Shapley, L. S. (1967), 'On balanced sets and cores', Naval Research Logistics Quarterly 14, 453-460.

Shapley, L. S. (1971), 'Cores of convex games', International Journal of Game Theory 1, 11-26.

Sharkey, W. (1982), 'Cooperative games with large cores', International Journal of Game Theory 11, 175182.

Van Gellekom, J. R. G., Potters, J. A. M. and Reijnierse, J. H. (1999), 'Prosperity properties of tu-games', International Journal of Game Theory 28, 211-227.

Van Velzen, B. (2005), Cooperation in Networks and Scheduling, PhD thesis, Tilburg University. I.S.B.N. 90-5668-147-8.

Van Velzen, B., Hamers, H. and Solymosi, T. (2008), 'Core stability in chain-component additive games', Games and Economic Behavior 62, 116-139.

Von Neumann, J. and Morgenstern, O. (1944), Theory of Games and Economic Behavior, Princeton University Press, Princeton, New Jersey. 